\title{
Fermion and graviton in Dirac-Born-Infeld braneworld models
}

\author{
Esmaeil Mazani $^{1, \mathrm{a}}$, Ali Tofighi ${ }^{1, \mathrm{~b}}$, Masoumeh Moazzen Sorkhi ${ }^{2, \mathrm{c}}$ \\ ${ }^{1}$ Department of Physics, Faculty of Basic Sciences, University of Mazandaran, P.O. Box 47416-95447, Babolsar, Iran \\ ${ }^{2}$ Department of Physics, Kosar University of Bojnord, Bojnord, Iran
}

Received: 30 July 2019 / Accepted: 11 March 2020 / Published online: 24 March 2020

(C) The Author(s) 2020

\begin{abstract}
In this paper, we study the braneworld models with non-standard kinetic term of Dirac-Born-Infeld (DBI) type in two case which are (I) in usual gravity and (II) in $f(T)$ gravity. In each of these cases, we examine gravity localization on branes and show that both models are stable and capable of gravity localizing in a similar manner to the standard case of braneworld models. In addition, we investigate the problem of fermion localization in both models and by considering the Yukawa coupling as a function of the warp factor, we show that the massless zero mode of fermion fields are localized on both types of the the branes. Meanwhile, the effect of parameters in both models is addressed on the zero mode and massive mode of graviton and fermion and the effective potentials. Finally, we study the gauge hierarchy problem in both models and we show that by choosing the appropriate parameters, both models are able to solve the gauge hierarchy problem between the Tev scale and the Planck scale.
\end{abstract}

\section{Introduction}

The braneworld models are an interesting subject in theoretical physics. These models were introduced for the first time in Ref. [1] as domain walls embedded in extra dimension and can provide creative ways to solve some problems in modern physics, including Casimir force [2] and the hierarchy problem [3]. Randall and Sundrum introduced another model called the R-S model, which did not require large extra dimensions to solve the hierarchy problem [4]. In their model, the brane was assumed to be extremely thin, and the size of extra dimension was not determined by the dynamics of the model. Goldberger and Wise [5], suggested an alternative mechanism that the size of extra dimension was stabilized by

\footnotetext{
a e-mail: e.mazani@stu.umz.ac.ir (corresponding author)

be-mail: a.tofighi@umz.ac.ir

c e-mail: m.m.sorkhi@kub.ac.ir
}

a five dimensional bulk scalar field with usual dynamics that could interact with gravity minimally [6] or non-minimally $[7,8]$. In their scenario, the brane is generated by a scalar field with kink configuration and it provides a domain wall that can be considered as non-singular versions of R-S model [9]. Recently, the standard braneworld scenario has been modified in such a way that the standard gravity action is extended to include scalar fields with generalized dynamics [10]. In these models, the Lagrange density has a non-standard form $L=K(X)-V(\phi)$, that is, the kinetic term is expressed as a non canonical form $K(X)$ which is a function of $X$. These theories are called $\mathrm{K}$-fields and were used for thick branes in Refs. [11-15]. The authors of Ref. [10] have considered two specific forms for $K(X)$, which are $K(X)=-X^{2} / 2$ and $K(X)=X+\alpha|X| X$, and with the help of first order formalism, they showed that their model is stable and capable of localizing gravity in a similar way to the standard case. An important example of K-field theories is DBI model, in which the kinetic term is of Dirac-Born-Infeld (DBI) type. The main difference between the DBI models and the models described above is the non-linear structure of the kinetic energy term. These models are also considered in the field of dark energy, so that in the framework of string theory, the accelerated expansion of the early universe, or the inflation problem, is explained by a model called DBI inflation [16-19]. On the other hand, the braneworld models with nonstandard kinetic term of DBI type have been investigated in Ref. [20] and it has been shown that these models have linear stable solutions.

The issue of localization of matter fields on different branes can be of great importance, since it reflects the fact that which brane structure is more acceptable from the phenomenological point of view. We know that the zero mode of massless scalar fields and graviton are localized on branes of different types [21-24], but for vector and Kalb-Ramond tensor fields, this does not happen [25,26], so other methods have been used to localize them $[9,27]$. On the other hand, in order to localize the fermions, we need to introduce the 
Yukawa coupling between the fermions and the background scalar fields [28-30], but if the background scalar field is an even function of extra dimension, then the Yukawa coupling mechanism does not work. One of the methods used to solve this problem in Ref. [31] is the introduction of a derivative fermion-scalar coupling in which the background scalar field can be an even or odd function of extra dimension. Another method is the introduction of a coupling between the spinor field and the scalar curvature of space-time, which is used in Ref. [32]. In general, the issue of the localization of different matter fields in braneworld models has been widely studied. For a complete review, you can see Refs. [33-37].

From another standpoint, braneworld models can be applied to gravitational theories, such as $f(T)$ gravity. The braneworld models in $f(T)$ gravity have been widely studied in Refs. [38-40]. On the other hand, the authors of Ref. [41] used the K-fields as the background scalar fields and by considering some specific forms for $f(T)$ and $K(X)$, they have shown that their solutions are stable, and the zero mode of graviton can be localized on the brane.

The K-field theories of DBI type are of particular importance because DBI models play an important role in inflationary models based on string theory, in which the inflation is considered as D-brane moving on the space-time manifold, in other words, inflation is described as the open string mode [42-44]. Therefore, the study of braneworld models with the kinetic energy term of DBI type (DBI braneworld models) can have interesting results. As has been said earlier, this issue has been recently considered by some authors, but the problem of graviton and fermion localization, both in the absence of $f(T)$ gravity and in $f(T)$ gravity, has not yet been investigated. In this paper, we intend to study the DBI braneworld models in two cases, which are: (1) DBI braneworld models in usual gravity, (2) DBI braneworld models in $f(T)$ gravity. In each of these cases, we examine the stability of the model and the graviton and fermion localization conditions. It should be noted that we use the derivative coupling term as Yukawa coupling and by presenting the mass-independent potential shapes in the corresponding Schrodinger-like equations, we examine the localization of bulk fermion and graviton fields on both types of branes mentioned above.

On the other hand, solving the hierarchy problem was one of the most important goals for proposing the small extra dimensional models, in particular the Randall-Sundrum model. So we were interested in exploring the hierarchy problem for the DBI braneworld models as well. To investigate this, we used a perturbation method in which the nonstandard term of kinetic energy is considered as a perturbation to the standard scenario.

The plan of this paper is as follows. In Sect. 2, we briefly summarize two braneworld models. To do this, we follow the approach of Ref. [10], for the first model and the approach of Ref. [41], for the second model. In Sect. 3, we study the localization of graviton field in these models. In Sect. 4, we study the localization of fermion field in these models. To do this, we assume the Yukawa coupling term as a function of the warp factor and the normalization condition of the fermion zero mode is discussed for this coupling. There is also a qualitative discussion of massive mode in Sect. 5. Section 6 is devoted to solving the hierarchy problem in both models and finally, a summary and conclusion are given in Sect. 7.

\section{DBI braneworld models}

In this section, we will investigate the braneworld models with non-standard kinetic term in which the kinetic term is of Dirac-Born-Infeld (DBI) type. We call these models as DBI braneworld models. We examine these models in two separate categories, which are (I) DBI braneworld model in usual gravity and (II) DBI braneworld model in $f(T)$ gravity. In the first model, we follow the approach outlined in Ref. [10] and we find the solutions of the model with the help of the first order formalism method. But in the second model, we follow the approach of Ref. [41], where another method is used instead of the usual first order formalism method. It should be noted that the first order formalism has been used in Refs. [38,39] for braneworld models with standard kinetic term in $f(T)$ gravity and braneworld models with non-standard kinetic term in $f(T)$ gravity with the specific form $K(X)=X+\alpha\left[(1+b X)^{n}-1\right]$, respectively. But in both cases, only the particular form of $f(T)=T+\alpha T^{n}$ was used. Thus, in order to consider more general forms of $f(T)$ and $K(X)$, the authors of Ref. [41] have used another method other than the first order formalism. In the following, we study both of the DBI braneworld models mentioned above.

\subsection{DBI braneworld models in usual gravity}

In this model, we use a brane with generalized dynamics where the five dimensional action is written in such a way that gravity is coupled to the scalar field in the following form [10],

$S=\int d^{5} x \sqrt{|g|}\left(-\frac{1}{4} R+L(\phi, X)\right)$,

where $R$ is the scalar curvature and we have $L(\phi, X)=$ $K(X)-V(\phi)$ in which $K(X)$ and $V(\phi)$ are the non-standard kinetic term and potential, respectively. The non-standard kinetic term $K(X)$ is a function of $X$ and in the standard case we have $K(X)=X$. The quantity $X$ is defined as $X=$ $-\frac{1}{2} g^{M N} \partial_{M} \phi \partial_{N} \phi$. On the other hand, the warped metric for a five dimensional space-time has the following general form,

$d s^{2}=g_{M N} d x^{M} d x^{N}=e^{2 A(y)} g_{\mu \nu} d x^{\mu} d x^{\nu}-d y^{2}$, 
where $e^{2 A(y)}$ is the warp factor and $g_{\mu \nu}$ is the metric of four dimensional curved brane with the signature $(+,-,-,-)$. Also we have $M, N=0,1,2,3,4$ and $\mu, v=0,1,2,3$. As usual we suppose that the scalar field $\phi$ and the warp factor $A$ only depend on the extra dimension $y$. That is, it can be said that $\phi=\phi(y)$ and $A=A(y)$, so we can obtain the equation of motion for the scalar field as follows,

$\left(K_{X}+2 X K_{X X}\right) \phi^{\prime \prime}-V_{\phi}=-4 K_{X} \phi^{\prime} A^{\prime}$.

Also, with respect to the metric (2), the Einstein equations are as follows,

$$
\begin{aligned}
A^{\prime \prime} & =\frac{4}{3} X K_{X}, \\
A^{\prime 2} & =\frac{1}{3}\left(K-2 X K_{X}-V\right),
\end{aligned}
$$

where prime denotes derivative with respect to $y$ and we use the notation $K_{X}=\frac{\partial K}{\partial X}$ and $K_{X X}=\frac{\partial^{2} K}{\partial X^{2}}$. It should be noted that for the static solutions, we have $X=-\frac{1}{2} \phi^{\prime 2}$. Now we apply the first order formalism to the braneworld scenario. This method was first introduced in Ref. [45] and it uses a function $W(\phi)$ called superpotential in such a way that

$A^{\prime}=-\frac{1}{3} W(\phi)$.

The Eqs. (4) and (5) in the first order formalism can be written as

$$
\begin{aligned}
K_{X} \phi^{\prime} & =\frac{1}{2} W_{\phi}, \\
K-2 K_{X} X-V(\phi) & =\frac{1}{3} W^{2},
\end{aligned}
$$

where $W_{\phi}$ denotes $W_{\phi}=\frac{\partial W}{\partial \phi}$. In this paper we would like to consider the Dirac-Born-Infeld (DBI) model in which the non-standard kinetic term $K(X)$ has the following form [46,47],

$K(X)=-a^{2}\left(\sqrt{1-\frac{2 X}{a^{2}}}-1\right)$,

where $a$ is a real parameter and it can control the high order powers in the covariant derivative. It can be shown that for $a \gg 1$, the kinetic term $K(X)$ returns to the standard case $K(X)=X$. Now, if we put relation (9) into equation (7), we arrive at the following equation for the DBI braneworld models,

$\phi^{\prime}=\frac{W_{\phi} a}{\sqrt{4 a^{2}-W_{\phi}^{2}}}$.

The Eqs. (6) and (10) must be solved together to obtain the scalar field and warp factor solutions, but before that, we must introduce the superpotential function $W(\phi)$. According to Ref. [20], we can consider the following two forms for superpotential,

$$
\begin{gathered}
W_{\phi}=\frac{1-\phi^{2}}{\sqrt{1+\frac{1}{a^{2}}\left(1-\phi^{2}\right)^{2}}}, \\
W_{\phi}=\frac{1-\sinh ^{2}(\phi)}{\sqrt{1+\frac{1}{a^{2}}\left(1-\sinh ^{2}(\phi)\right)^{2}}} .
\end{gathered}
$$

We first consider the first form for superpotential to solve the equations (6) and (10), but since the analytical solutions of these equations are difficult, we solve them numerically. By numerically solving of these equations, the graphs for the scalar field $\phi(y)$ and the warp factor $A(y)$ are obtained for different values of parameter $a$. These diagrams are shown in Fig. 1a, b. Our calculations show that the results for the second superpotential are similar to the first one. Therefore, in the entire paper, only the first form of the superpotential will be considered.

On the other hand, according to the Eq. (8), the scalar field potential can be obtained as follows,

$V(\phi)=a^{2}\left(1-\frac{1}{G(\phi)}\right)-\frac{1}{3} W(\phi)^{2}$

where $G(\phi)$ has the following form,

$G(\phi)=2 \sqrt{\frac{\phi^{4}-2 \phi^{2}+a^{2}+1}{3 \phi^{4}-6 \phi^{2}+4 a^{2}+3}}$

and we have $W(\phi)=\int W_{\phi} d \phi$. By calculating $W(\phi)$, which appears as an elliptic function, we can plot the shape of the scalar potential function $V(\phi)$. In Fig. 1c, we plotted this potential for different values of the parameter $a$. It can be seen that the potential is stable for different values of this parameter. Furthermore, the stable values $(\langle\phi\rangle)$ are independent of the parameter $a$, but by increasing the parameter $a$ and approaching the standard scenario, the minimum value of the potential $(V(\langle\phi\rangle))$ gets larger negative values. This means that the $V(\langle\phi\rangle)$ is proportional to the parameter $a$. It should be noted that the above potential shape corresponds to the scalar field potential of the braneworld models with non-standard kinetic term $K(X)=X+\alpha|X| X$, which is studied in Ref. [10].

\subsection{DBI braneworld models in $f(T)$ gravity}

In this section, we follow the approach outlined in Ref. [41] and study the DBI braneworld models in $f(T)$ gravity. According to $f(T)$ gravity in braneworld models, we have to replace the torsion scalar $T$ with $f(T)$ which is a function of $T$. It should be noted that the torsion scalar can be calculated 


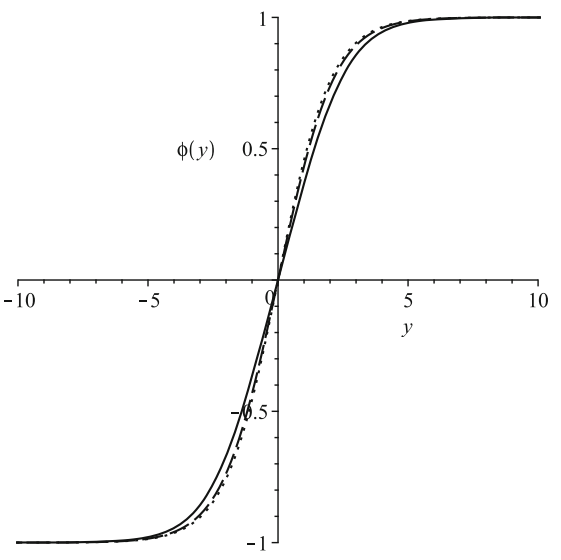

(a)

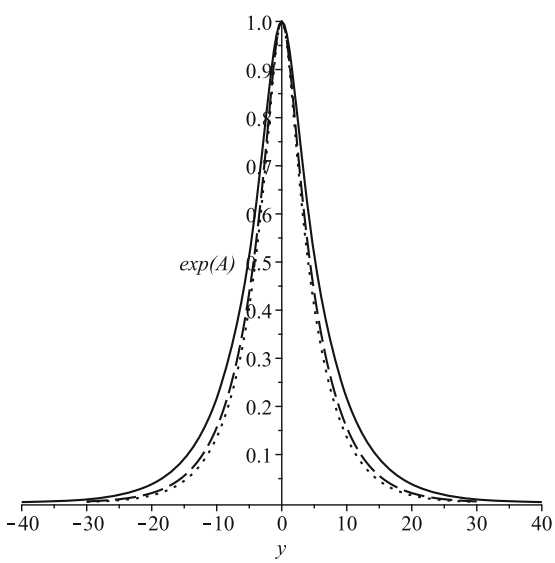

(b)

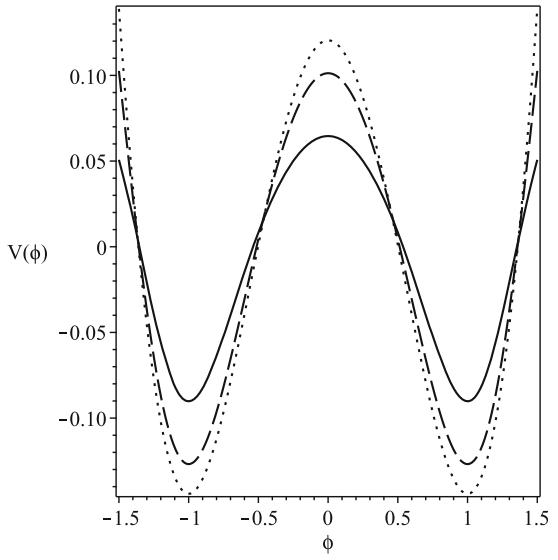

(c)

Fig. 1 The profile of a the background scalar field $\phi(y), \mathbf{b}$ the warp factor $e^{A(y)}$ and $\mathbf{c}$ the scalar field potential $V(\phi)$ for $a=1$ (solid line), $a=2$ (dashed line), $a=5$ (dotted line)

as $T=-12 A^{\prime 2}$. The total action in these branworlds models has the following form,

$S=-\frac{1}{4} \int d^{5} x \operatorname{ef}(T)+\int d^{5} x L_{M}$

where $L_{M}=e(K(X)-V(\phi))$ is the Lagrangian of the background scalar field and $e$ is the determinant of vielbein. Also, the metric of five-dimensional space-time is the same as the relation (2). With respect to action (15) and the ansatz (2), the equations of motion are obtained as follows,

$\frac{1}{4} f+\frac{1}{2} f_{T}\left(3 A^{\prime \prime}+12 A^{\prime 2}\right)-36 f_{T T} A^{\prime 2} A^{\prime \prime}=K-V$,

$\frac{1}{4} f+6 f_{T} A^{\prime 2}=K-V+K_{X} \phi^{\prime 2}$,

$4 A^{\prime} \phi^{\prime} K_{X}+\partial_{y}\left(K_{X} \phi^{\prime}\right)=V_{\phi}$,

where we used the notation $f_{T}=\frac{\partial f}{\partial T}$ and $f_{T T}=\frac{\partial^{2} f}{\partial T^{2}}$, and the prime denotes the derivative with respect to $y$. Among the Eqs. (16)-(18), only two of them are independent, so we focus only on Eqs. (16) and (17). If we subtract Eq. (17) from Eq. (16), we arrive at

$\frac{3}{2} f_{T} A^{\prime \prime}-36 f_{T T} A^{\prime 2} A^{\prime \prime}=-K_{X} \phi^{\prime 2}$,

which this equation can be written as the following form,

$\frac{3}{2} \partial_{y}\left(f_{T} A^{\prime}\right)=-K_{X} \phi^{\prime 2}$

This equation helps us to find solutions to our braneworld model. In our model, the kinetic energy term is of the DBI type. Therefore, if we put relation (9) in equation (20), the equation of DBI braneworld model in $f(T)$ gravity is obtained as follows,

$\frac{3}{2} \partial_{y}\left(f_{T} A^{\prime}\right)=-\frac{\phi^{\prime 2}}{\sqrt{1+\frac{\phi^{\prime 2}}{a^{2}}}}$.

To solve Eq. (21), we need to introduce the function $f(T)$ that must have the necessary conditions for gravity localization. For example, in Ref. [38], the function $f(T)$ was considered as $f(T)=T+\alpha T^{n}$, and with the help of the first order formalism, they were able to solve these models and studied the localization of matter fields. In this paper, we use the following three forms for $f(T)$. The first two cases were introduced in Ref. [41], but the third one was introduced by ourselves,

$f_{T}=\exp \left(\frac{T}{T_{0}}\right)$

$f_{T}=\sum_{n=0}^{N} \alpha_{n} T^{n}$,

$f_{T}=-\ln \left(\frac{1+T}{2-T}\right)$,

where we have $T_{0}=24 k^{2} m^{2}$, in which $k$ and $m$ are arbitrary constants. Since we need only the warp factor to study the zero mode of graviton and fermion, we consider the background scalar field as a constant function as follows,

$\phi(y)=m \tanh (k y)$.

Now, with respect to the background scalar field $\phi(y)$, we can solve the Eq. (21) for different forms of the function $f_{T}$. It is difficult to solve this equation by analytical methods, so we solve it numerically for different forms of $f_{T}$. For 


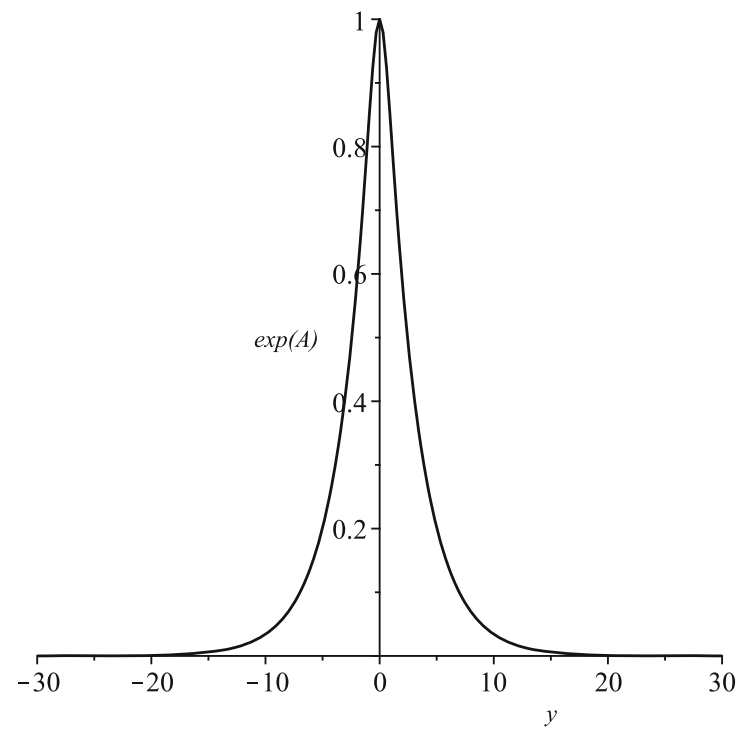

(a)

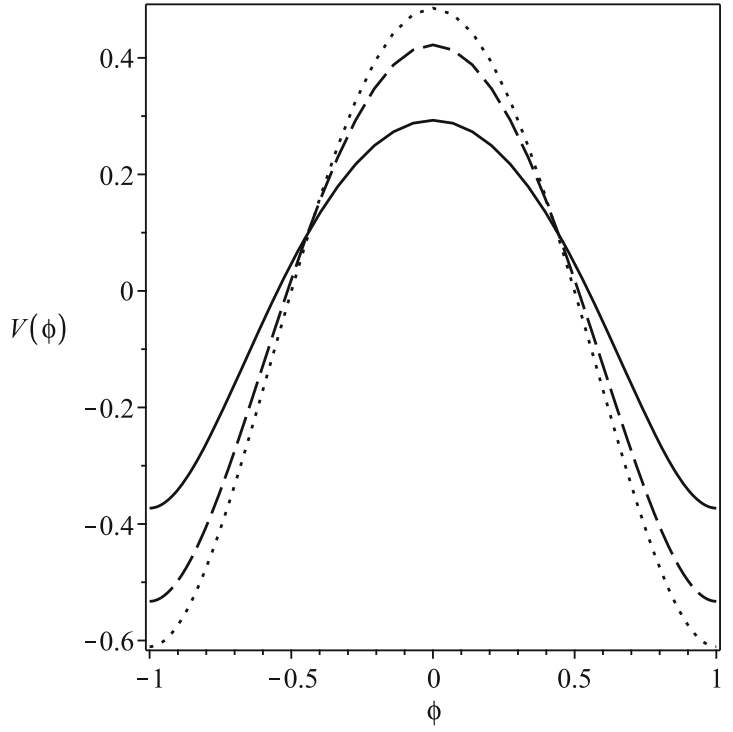

(b)

Fig. 2 The profile of a the warp factor $e^{A(y)}$ for $a=1, k=m=1$ and $\mathbf{b}$ the scalar field potential $V(\phi)$ for $a=1$ (solid line), $a=2$ (dashed line), $a$ $=5($ dotted line $)$

example, the shape of the warp factor for $f_{T}=\exp \left(T / T_{0}\right)$ is obtained as Fig. 2a. Finally, it should be noted that the scalar field potential $(V(\phi))$ is computable from the Eq. (17). Our numerical calculations show that this scalar field potential is stable and its diagram is generally similar to the scalar field potential diagram in the first model. In Fig. 2b, the scalar field potential in the second model for $f_{T}=\exp \left(T / T_{0}\right)$ is plotted. As we see, the stable values $(\langle\phi\rangle= \pm m)$ are independent of the parameter $a$, but by increasing the parameter $a$ and approaching the standard scenario, the minimum value of the potential $(V(\langle\phi\rangle))$ gets larger negative values. Therefore the effect of parameter $a$ on the scalar field potential in the second model is similar to the effect of this parameter in the first model (see Fig. 1c).

\section{Localization of graviton}

In this section, we would like to investigate the localization of graviton in DBI braneworld models. In order to obtain the zero mode of graviton in the first model, we follow the approach of Ref. [10] in which by considering the metric perturbations and the first order contributions of Einstein equations, we can arrive at the schrodinger-like equation and the zero mode of graviton. But in the second model, we follow the approach of Ref. [41], in which the tensor perturbation method was used. By considering the transverse-traceless tensor perturbation which is related to the four dimensional gravitons, the perturbation equation can be obtained and with the help of the perturbation equation, we can arrive at the schrodinger-like equation for the extra dimensional profile.
In the following, we study the localization of graviton zero mode in both DBI braneworld models.

\subsection{Localization of graviton on DBI braneworld models in} usual gravity

It can be shown that Einstein's equations for braneworld models with generalized dynamics are simplified as follows [10],

$\left(\partial_{y}^{2}+4 A^{\prime} \partial_{y}-e^{-2 A} \square\right) \bar{h}_{\mu \nu}=0$,

where $\square$ is d'Alembert operator. Now, if we use the relation $d z=e^{-A(y)} d y$ and the change of variables $H_{\mu \nu}=e^{-i p x+3 / 2 A(z)} \bar{h}_{\mu \nu}$, finally we arrive at the following Schrodinger-like equation,

$\left[-\partial_{z}^{2}+U(z)\right] H_{\mu \nu}=p^{2} H_{\mu \nu}$

where Schrodinger-like potential $U(z)$ is

$U(z)=\frac{9}{4} A^{\prime 2}(z)+\frac{3}{2} A^{\prime \prime}(z)$.

Since analytical solution of the warp factor is not available, therefore, an analytic analysis of the potential function is not possible, so we will consider its numerical analysis. To do this, with the help of the relation $d z=e^{-A(y)} d y$, we write the potential in the $y$ coordinate as follows:

$$
\begin{aligned}
U(y)= & \frac{9}{4}\left[e^{A(y)} \partial_{y} A(y)\right]^{2} \\
& +\frac{3}{2} e^{2 A(y)}\left[\left(\partial_{y} A(y)\right)^{2}+\partial_{y}^{2} A(y)\right] .
\end{aligned}
$$


Using the relation $U(y)$, we can obtain the shape of the potential function. In this paper, we would like to examine the effect of parameter $a$, so we plot the shape of potential for different values of this parameter in Fig. 3a. The potentials tend to zero at the infinity so they are volcano-like. On the other hand, increasing the parameter $a$ causes the minima and maxima of the potential increase, which means that the ability to gravity localization is proportional to the parameter a.

The Eq. (27) can be written as follows,

$$
\left(\partial_{z}+\frac{3}{2} A^{\prime}(z)\right)\left(-\partial_{z}+\frac{3}{2} A^{\prime}(z)\right) H_{\mu \nu}=p^{2} H_{\mu \nu} .
$$

This equation shows that the graviton does not have a boundary state with a negative mass. In addition, if we put $p=0$, we conclude that the zero mode of graviton is proportional to

$\Psi_{0}(z) \propto e^{\frac{3}{2} A(z)}$,

where $\Psi_{0}(z)$ denotes the graviton zero mode. In order to localize graviton on the brane, we should have $\int \Psi_{0}^{2}(z) d z<$ $\infty$. Therefore, the necessary condition for the localization of graviton zero mode is

$$
\int \Psi_{0}^{2}(z) d z \propto \int e^{3 A(z)} d z<\infty
$$

According to our numerical calculations, the limit of the warp factor when $z \longrightarrow \pm \infty$, is equal to $(-\infty)$ and since the integrand in Eq. (32) is a continuous function, it can be concluded that the result of the integral is finite and the localization condition is established. Now, with respect to relation (31), we can plot the zero mode of graviton for different values of the parameter $a$ as Fig. $3 \mathrm{~b}$. It can be seen that the zero mode of graviton is localized on the brane, and by increasing the parameter $a$, its localization is strengthened. These results are in agreement with the results of changing the parameter $a$ on the potential function that we discussed earlier.

\subsection{Localization of graviton on DBI braneworld models in $f(T)$ gravity}

Using the approach outlined in Ref. [41] and with the tensor perturbation method, the tensor perturbation equation is obtained as follows,

$\left(\partial_{z}^{2}+2 H \partial_{z}+\square^{(4)}\right) \gamma_{\mu \nu}=0$,

where $\square^{(4)}=g^{\mu \nu} \partial_{\mu} \partial_{\nu}$ and we have

$H=\frac{3}{2} \partial_{z} A+12 e^{-2 A}\left(\left(\partial_{z} A\right)^{3}-\partial_{z}^{2} A \partial_{z} A\right) \frac{f_{T T}}{f_{T}}$.
Now, if we use the KK decomposition, we have

$\gamma_{\mu \nu}\left(x^{\rho}, z\right)=\epsilon_{\mu \nu}\left(x^{\rho}\right) F(z) \Psi(z)$,

where we have

$F(z)=e^{-\frac{3}{2} A(z)+\int k(z) d z}$,

and

$k(z)=12 e^{-2 A}\left(\partial_{z}^{2} A \partial_{z} A-\left(\partial_{z} A\right)^{3}\right) \frac{f_{T T}}{f_{T}}$.

By putting relation (35) in Eq. (33), we arrive at two independent equations, which are: (1) The Klein-Gordon equation for the four-dimensional KK gravitons,

$\left(\square^{(4)}+m^{2}\right) \epsilon_{\mu \nu}\left(x^{\rho}\right)=0$,

(2) The Schrodinger-like equation for the extra dimension profile,

$\left(-\partial_{z}^{2}+U(z)\right) \Psi(z)=m^{2} \Psi(z)$,

where $m$ is KK graviton mass and the effective potential $U(z)$ has the following form,

$U(z)=H^{2}+\partial_{z} H$.

The Schrodinger-like equation (39) can be written as follows,

$\left(-\partial_{z}+H\right)\left(\partial_{z}+H\right) \Psi(z)=m^{2} \Psi(z)$.

This equation shows that there is no four-dimensional graviton with $m^{2}<0$, so each solution is stable for the branes in $f(T)$ gravity.

In Eq. (39), if we put $m=0$, the zero mode of fourdimensional massless graviton is obtained as follows,

$\Psi_{0}(z)=N_{0} e^{\frac{3}{2} A(z)-\int k(z) d z}$,

where $N_{0}$ is the normalization coefficient. In order to localize graviton on the brane, we should have $\int \Psi_{0}^{2}(z) d z<\infty$. Therefore, the necessary condition for the localization of graviton zero mode is

$\int \Psi_{0}^{2}(z) d z=\int N_{0}^{2} e^{3 A(z)-2 \int k(z) d z}<\infty$.

Numerical calculations with any of the above-mentioned $f(T)$ show that in Rel. (43) the integrand in infinity tends to zero, which means that the result of the integral is finite and the localization condition is established. Now, with respect 


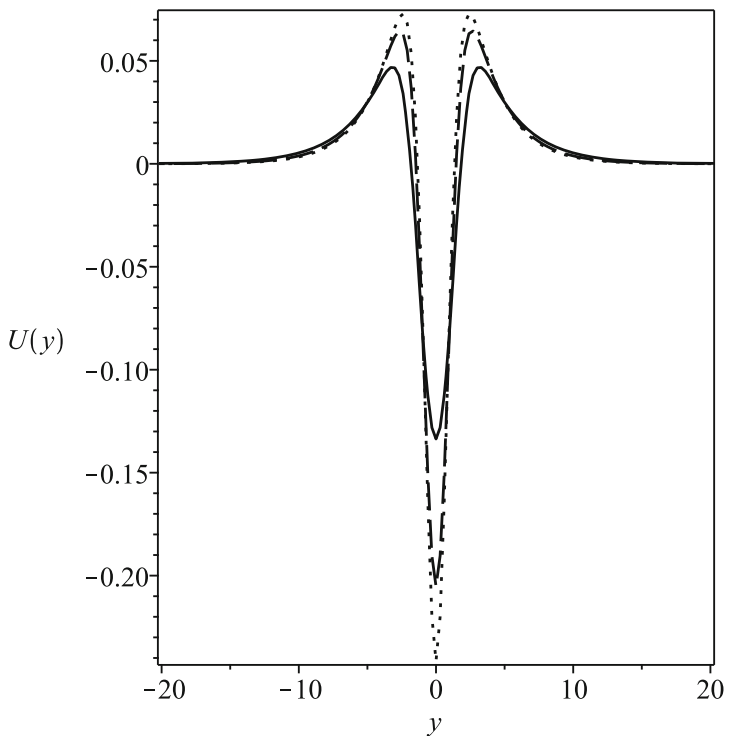

(a)

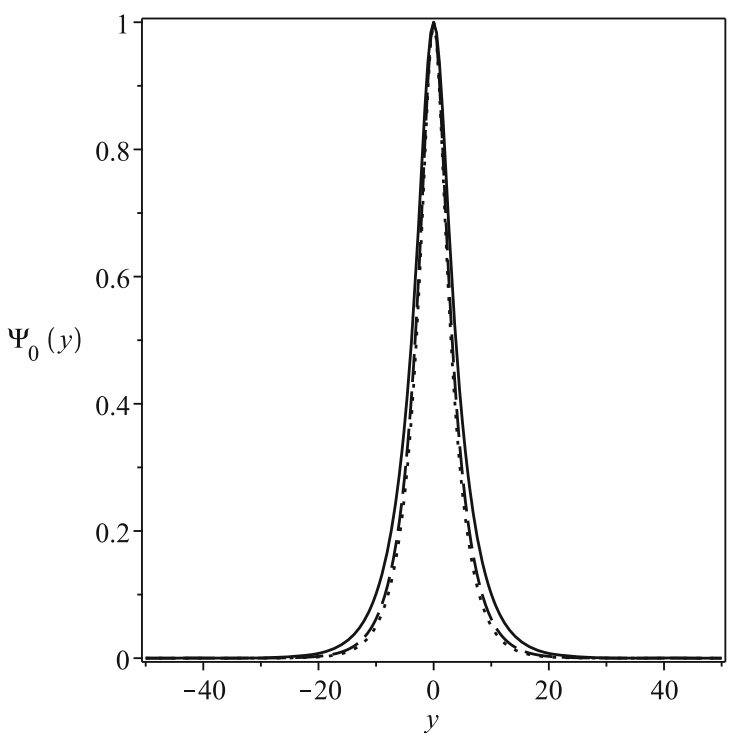

(b)

Fig. 3 The profile of $\mathbf{a}$ the potential $U(y)$ and $\mathbf{b}$ the zero mode of graviton $\Psi_{0}(y)$ for $a=1$ (solid line), $a=2$ (dashed line), $a=5$ (dotted line)

to the Eq. (40) and using the relation $d z=e^{-A(y)} d y$, we can plot the shapes of effective potentials $U(y)$ for different forms of the function $f_{T}$ as Fig. 4. Our investigations show that all potentials tend to zero at infinity and they are volcano-like but there are differences between them. For $f_{T}=\exp \left(T / T_{0}\right)$, the potential always has a negative well at the origin and for $f_{T}=-\ln \left(\frac{1+T}{2-T}\right)$, the potential always has two negative wells on both sides of the origin. But for $f_{T}=\sum_{n=0}^{N} \alpha_{n} T^{n}$, both states can occur, that is, there are two wells in the two sides of the origin as Fig. $4 \mathrm{~b}$, or the parameters $\alpha$ may be chosen in a way that the potential only has a negative well at the origin. On the other hand, as we see in Fig. $4 \mathrm{a}$, when the parameter $a$ becomes larger, the values of two maxima of the potential increase. This is an interesting feature, because it changes the behavior of the massive modes in the region between the two maxima where our fourdimensional universe is located. Meanwhile, with increasing the parameter $a$, the depth of well for all three forms of the function $f_{T}$ increases, but the thickness of the well does not change significantly. Thus, the ability of the effective potential in trapping the graviton is proportional to the parameter a.

Now, we would like to investigate the localization of graviton. In the following, we consider the zero mode of graviton for three different types of $f(T)$,

Type (I) $f(T)=\exp \left(\frac{T}{T_{0}}\right)$ : In this case, taking into account the relation (42) and the numerical solution of warp factor obtained in Sect. 2.2, we can plot the zero mode of graviton for different values of the parameter $a$. As shown in Fig. 5a, the zero mode is localized on the center of the brane and with increasing parameter $a$, the graviton localization is strengthened.

Type (II) $f_{T}=\sum_{n=0}^{N} \alpha_{n} T^{n}$ : In this case, if $N=1$, we have $f_{T}=\alpha_{0}+\alpha_{1} T$ and the zero mode of graviton can be plot as Fig. 5b for different values of the parameter $a$. As we see the zero mode of graviton can be localized between the two sub-branes. It should be noted that in this case, by changing the parameters $\alpha_{0}$ and $\alpha_{1}$, there may be a situation where the zero mode is localized on the center of brane. To investigate this, we assume that $\alpha_{0}=1$, so that the form of the function $f(T)$ is obtained as follows,

$f(T)=T+\frac{1}{2} \alpha_{1} T^{2}$,

considering this relation for $f(T)$, we would like to examine the effect of parameter $\alpha_{1}$ on the zero mode of graviton. Our investigations show that, as shown in Fig. 6a, when $\alpha_{1}$ tends to zero, the effective potential which has two wells at the sides of the origin, changes to a well at the origin. Also, according to Fig. 6b, the zero mode instead of being localized between the two sub-branes is localized on the center of brane. In other words, it can be said that by increasing the parameter $\alpha_{1}$, the brane is split into two sub-branes. This result is expected because it is consistent with Einstein's general relativity. The reason is that in relation (44), if $\alpha_{1}$ tends to zero, the sentence $\frac{1}{2} \alpha_{1} T^{2}$ can be ignored, and we have $f(T)=T$, which is the same as the general relativity scenario.

Now we want to go to the case $N=2$, and we consider $f_{T}$ as $f_{T}=\alpha_{0}+\alpha_{1} T+\alpha_{2} T^{2}$. In this case, if we plot the zero mode of graviton for different values of the parameters, we see that the zero mode is localized on the brane. Also, it 


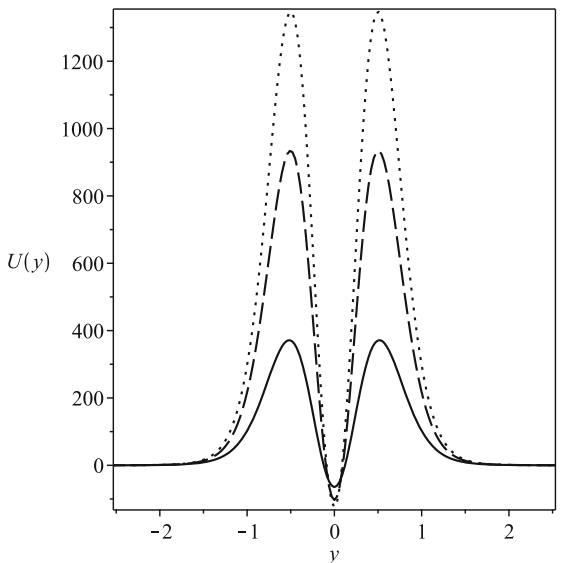

(a)

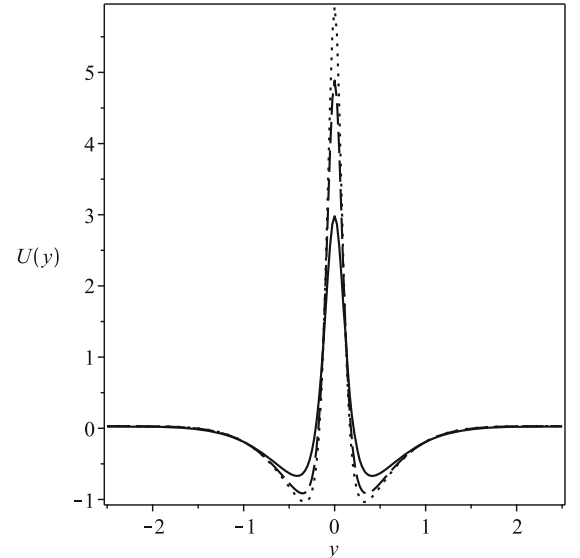

(b)

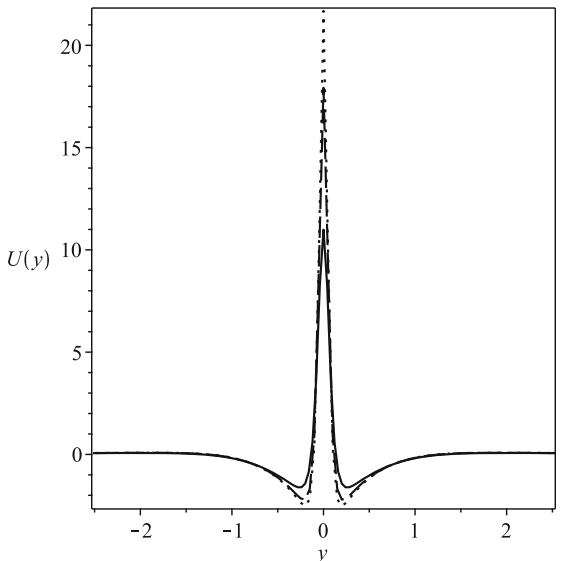

(c)

Fig. 4 The profile of the effective potentials for a $f_{T}=\exp \left(T / T_{0}\right), \mathbf{b} f_{T}=\alpha_{0}+\alpha_{1} T, \alpha_{0}=2, \alpha_{1}=-10$, and $\mathbf{c} f_{T}=-\ln \left(\frac{1+T}{2-T}\right)$. In all figures we set $a=1$ (solid line), $a=2$ (dashed line), $a=5$ (dotted line)

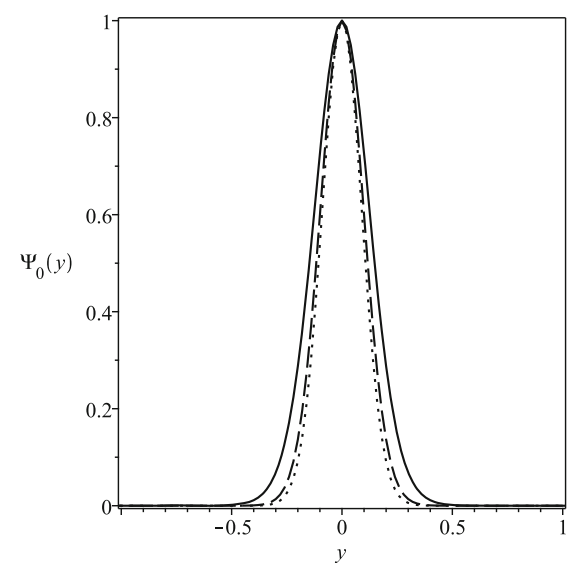

(a)

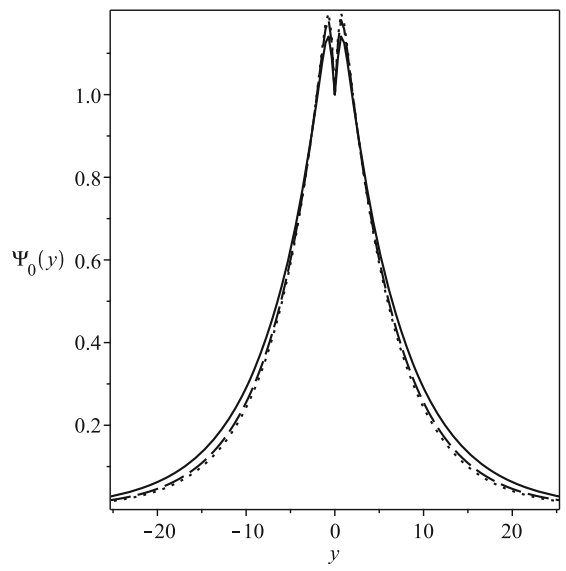

(b)

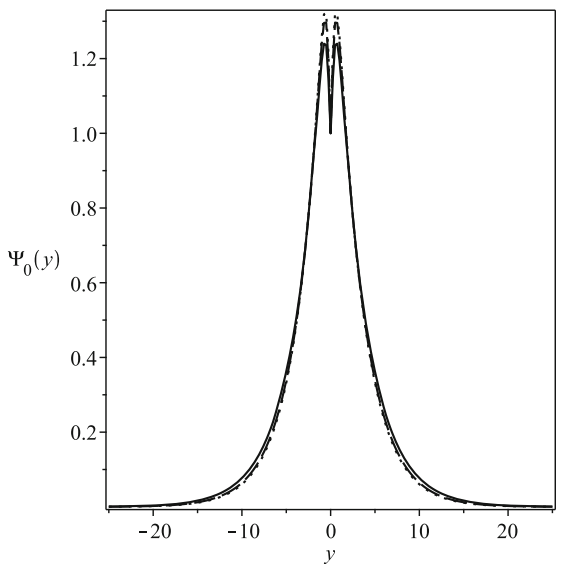

(c)

Fig. 5 The profile of graviton zero mode for a $f_{T}=\exp \left(T / T_{0}\right)$, b $f_{T}=\alpha_{0}+\alpha_{1} T, \alpha_{0}=2, \alpha_{1}=-10$ and $\mathbf{c} f_{T}=-\ln \left(\frac{1+T}{2-T}\right)$. In all figures we set $a=1$ (solid line), $a=2$ (dashed line), $a=5$ (dotted line)

was observed that if $\alpha_{0}=1$ and $\alpha_{1}$ and $\alpha_{2}$ tend to zero, then the zero mode of graviton has a peak that is consistent with general relativity. In the end of this section, we note that in all states $N=1, N=2$ and ..., increasing the parameter $a$ causes the localization of the zero mode of graviton to be strengthened.

Type (III) $f_{T}=-\ln \left(\frac{1+T}{2-T}\right)$ : In this case, we can plot the zero mode of graviton for different values of the parameter a. As shown in Fig. 5c, the zero mode is localized between the two sub-brane. On the other hand, It can be seen that by increasing the parameter $a$, the graviton localization is strengthened.

As the final point of this section, we note that in each of the three forms of $f_{T}$, the localization of graviton zero mode has its own characteristics. Studies show that for $f_{T}=\exp \left(T / T_{0}\right)$, the zero mode is always localized on the center of brane and for $f_{T}=-\ln \left(\frac{1+T}{2-T}\right)$, the zero mode is always localized between the two sub-branes but for $f_{T}=\sum_{n=0}^{N} \alpha_{n} T^{n}$, depending on the parameters $\alpha$, two different states may occur, the zero mode is localized on the center of brane, or the zero mode localization occurs between the two sub-branes. Therefore, the function $f_{T}$ plays an important role in the problem of graviton localization in the DBI braneworld models in $f(T)$ gravity.

\section{Localization of fermion}

In this section, we would like to investigate the localization of the bulk fermion fields by analyzing the potential of the corresponding Schrodinger-like equation for their KK modes. We know that the introduction of Yukawa coupling between the scalar and spinor field is necessary condition for the localization of fermions on a brane. The action of a massive bulk 


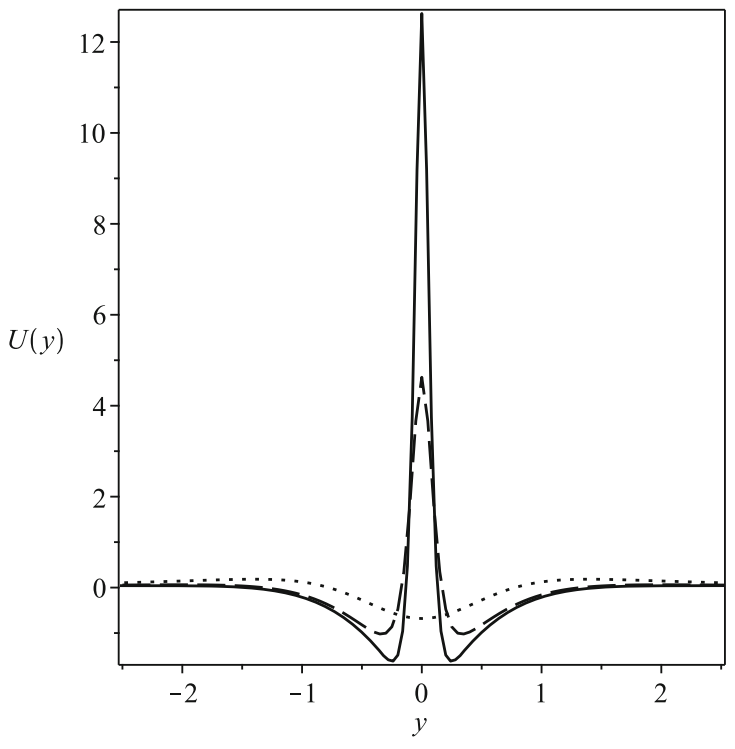

(a)

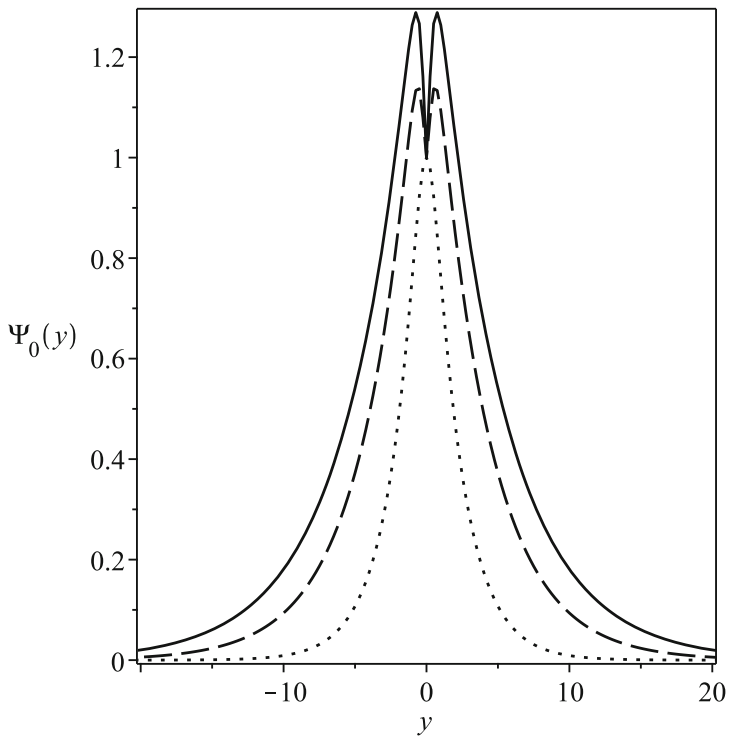

(b)

Fig. 6 The profile of a the effective potential and $\mathbf{b}$ the graviton zero mode for $f(T)=T+\frac{1}{2} \alpha_{1} T^{2}$ with $\alpha_{1}=-5$ (solid line), $\alpha_{1}=-2$ (dashed line), $\alpha_{1}=-0.01$ (dotted line)

fermion field can be written as the following form [48,49],

$S=\int d^{5} x \sqrt{-g}\left(\bar{\Psi} \Gamma^{M}\left(\partial_{M}+\omega_{M}\right) \Psi-\bar{\Psi} F(y) \Psi\right)$

where the $\omega_{M}$ is the spin connection and $F(y)$ is an odd function of the extra dimensional coordinate $y$. The metric for five-dimensional space-time is given by Eq. (2), but if we use the relation $d z=e^{-A(y)} d y$ and write it in the $z$ coordinate, the equation of motion for the fermion field can be obtained as

$\frac{1}{\sqrt{-g}}\left[\gamma^{\mu} \partial_{\mu}+\gamma^{5}\left(\partial_{z}+2 \dot{A}\right)-e^{A} F(y)\right] \Psi=0$,

where the dot denotes the derivative with respect to $z$. To solve the Eq. (46), we use the chiral decomposition as follows

$\Psi(x, z)=e^{-2 A} \sum\left(\psi_{L n}(x) f_{L n}(z)+\psi_{R n}(x) f_{R n}(z)\right)$,

where $\psi_{L n}(x)$ and $\psi_{R n}(x)$ are the left-handed and righthanded components of the four-dimensional spinor field respectively and they satisfy the four-dimensional Dirac equations. Also, $f_{L n}(z)$ and $f_{R n}(z)$ are the fermion KK modes and it can be shown that they satisfy the following schrodinger-like equations

$$
\begin{aligned}
& {\left[-\partial_{z}^{2}+V_{L}(z)\right] f_{L n}(z)=m_{n}^{2} f_{L n}(z),} \\
& {\left[-\partial_{z}^{2}+V_{R}(z)\right] f_{R n}(z)=m_{n}^{2} f_{R n}(z)}
\end{aligned}
$$

where the effective potentials $V_{L}$ and $V_{R}$ in the $y$ coordinate have the following forms,

$V_{L}(z(y))=\left(e^{A} F(y)\right)^{2}-e^{2 A}\left(A^{\prime} F(y)+F^{\prime}(y)\right)$,
$V_{R}(z(y))=\left(e^{A} F(y)\right)^{2}+e^{2 A}\left(A^{\prime} F(y)+F^{\prime}(y)\right)$.

In order to investigate the above effective potentials, we must have the warp factor $A(y)$ and the function $F(y)$. Since we have freedom to choose the Yukawa coupling, we consider it as a function of the warp factor $A(y)$, i.e., $F(y)=-\eta \partial_{y} A(y)$.

In Eqs. (48) and (49), if we set $m_{n}=0$, the zero mode of left-handed and right-handed fermions are obtained as follows,

$$
\begin{aligned}
& f_{L 0}(y) \propto \exp \left[-\int_{0}^{z} e^{A(\dot{z})} F(y) d z^{\prime}\right] \\
& =\exp \left[-\int_{0}^{y} F\left(y^{\prime}\right) d y^{\prime}\right], \\
& f_{R 0}(y) \propto \exp \left[+\int_{0}^{z} e^{A(\dot{z})} F(y) d z^{\prime}\right] \\
& =\exp \left[+\int_{0}^{y} F\left(y^{\prime}\right) d \dot{y}\right] .
\end{aligned}
$$

On the other hand, the normalization condition of the lefthanded fermion zero mode can be written as

$$
\int_{-\infty}^{\infty} d y \exp \left[-A(y)-2 \int_{0}^{y} F(\dot{y}) d \dot{y}\right]<\infty .
$$


Now, by setting $F(y)=-\eta \partial_{y} A$ in Eqs. (52) and (53), we get zero mode solutions as

$f_{L_{0}, R_{0}}(y) \propto \exp ( \pm \eta A(y))$,

and the normalization condition (54) can be written as

$\int_{-\infty}^{\infty} \exp [(2 \eta-1) A(y)] d y<\infty$

Our numerical calculations show that, in both DBI braneworld models, we have

$A(y \longrightarrow \pm \infty)=-\infty$,

Considering this asymptotic behavior of the warp factors in both models, it is easy to see that for $\eta>\frac{1}{2}$ the left-chiral zero mode can be localized in both types of DBI braneworld models.

\subsection{Localization of fermion on DBI braneworld models in usual gravity}

In the Sect. 2.1, we obtained the solutions of the warp factor and the background scalar field for the DBI braneworld models with numerical methods and we presented the graphs of $A(y)$ and $\phi(y)$ in Fig. 1. Now, with the help of the Eqs. (50) and (51), we can plot the effective potentials $V_{L, R}(y)$ for different values of the parameter $a$ as Fig. 7. As we see, the potential $V_{L}(y)$ is volcano-like which means that there is no mass gap to separate the zero mode from the excited modes. Also, the potential $V_{L}(y)$ has a negative well at the origin, so the left-handed fermion may have a massless mode but the potential $V_{R}(y)$ does not have a well structure and does not support the zero mode. On the other hand, by increasing the parameter $a$, the depth of the well increased, which means that the ability of the effective potential $V_{L}$ in trapping the fermions is proportional to the parameter $a$. At the beginning of this section, we see that with respect to the normalization condition (56), for $\eta>\frac{1}{2}$ the left-chiral zero mode can be localized on the brane. Now, with the help of Eq. (55), we can arrive at the zero mode of left-handed and right-handed fermions. We plot the zero mode of fermions as Fig. 8. It can be seen that the left-handed zero mode is localized on the center of the brane but for the right-handed zero mode, this does not happen which indicates that only one of the left and right handed fermion zero mode may be localized on the brane. This is consistent with the shapes of potentials $V_{L}$ and $V_{R}$ that we discussed earlier. On the other hand, it can be seen that by increasing the parameter $a$, the localization of left-chiral fermion zero mode is strengthened.
4.2 Localization of fermion on DBI braneworld models in $f(T)$ gravity

In Sect. 2.2, to obtain the warp factor, we considered the background scalar field as a constant function $\phi(y)=m \tanh (k y)$ and plotted the warp factor with numerical methods as Fig. 2a. Now, with the warp factor $A(y)$, we can use the Eqs. (50) and (51) to obtain the shapes of the effective potentials $V_{L}$ and $V_{R}$. As a result, we plot these potentials for $f(T)=\exp \left(T / T_{0}\right)$ as shown in Fig. 9. Again, it can be seen that the potential $V_{L}(y)$ is volcano-like and it has a negative well at the origin, so the left-handed fermion may have a massless mode but the potential $V_{R}(y)$ does not have a well structure and does not support the zero mode. Also, by increasing the parameter $a$, the depth of the well increased, which means that the ability of the effective potential $V_{L}$ in trapping the fermions is proportional to the parameter $a$. On the other hand, according to the normalization condition (56), for $\eta>\frac{1}{2}$ the left-chiral zero mode can be localized on the brane. Therefore, with the help of Eq. (55), we can plot the zero mode of left-handed and right-handed fermions as shown in Fig. 10. We can see that the left-chiral zero mode is localized on the brane but for the right-chiral zero mode, this does not happen. This is consistent with the shapes of potentials $V_{L}$ and $V_{R}$ that we discussed earlier. It should be noted that for each of three forms of the function $f_{T}$, the potential $V_{L}$ has a negative well at the origin and the zero mode of leftchiral fermion is always localized on the center of the brane. Also, it can be seen that by increasing the parameter $a$, the localization of left-chiral fermion zero mode is strengthened.

\section{A qualitative discussion on the graviton and fermion massive modes}

Now we would like to discuss the massive modes of graviton and fermion on both DBI braneworld models. To do this, we have to solve the equations (27), (39), (48) and (49) for $m_{n} \neq 0$. In some cases, the analytical solutions of these Schrodinger-like equations can be obtained. For example in Refs. [36,37], the analytical solutions of these equations are computed. In our work due to the more complex structure of the functions $W_{\phi}$ defined in relations (11) and (12), it is not possible to analytically solve the Schrodinger-like equations for massive modes. So, like the approach of Refs. [50-52], we solve these equations numerically. As we have seen in the Sects. 3 and 4, the effective potentials tend to zero at infinity. This means that the potentials provide no mass gap to separate the zero mode of graviton and fermion from the excited KK modes; i.e., there exists a continuous spectrum of KK modes for graviton as well as for fermion. On the other hand, the potentials are symmetric so the wave functions such as $f_{L n, R n}(z)$ can be either even or odd. Thus, we use 


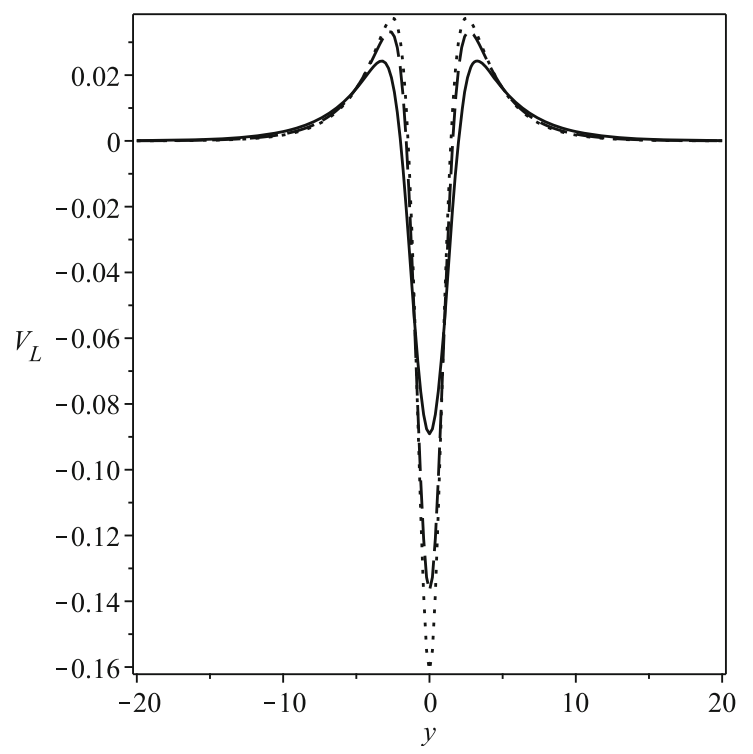

(a)

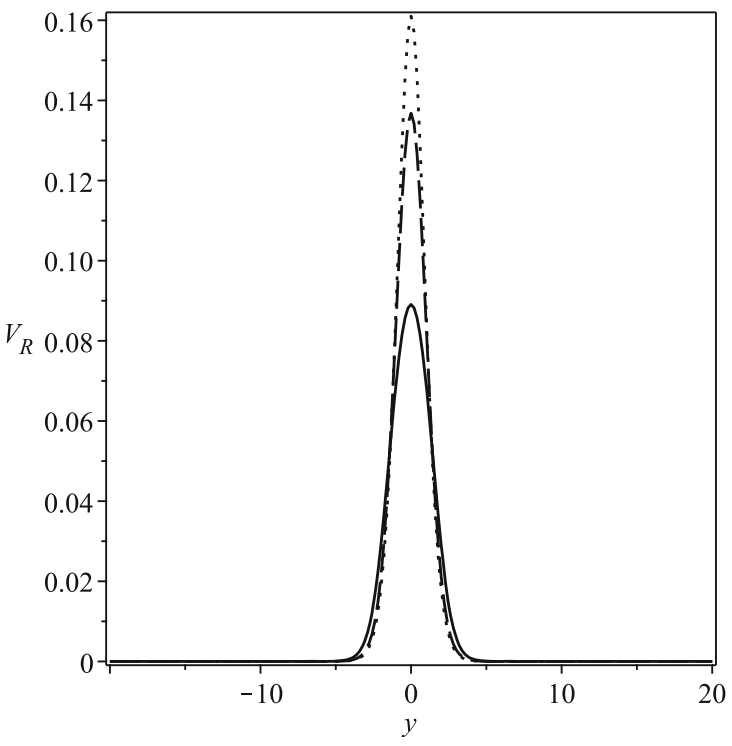

(b)

Fig. 7 The profile of the potentials $\mathbf{a} V_{L}(y)$ and $\mathbf{b} V_{R}(y)$ for $a=1$ (solid line), $a=2$ (dashed line), $a=5$ (dotted line), $\eta=1$

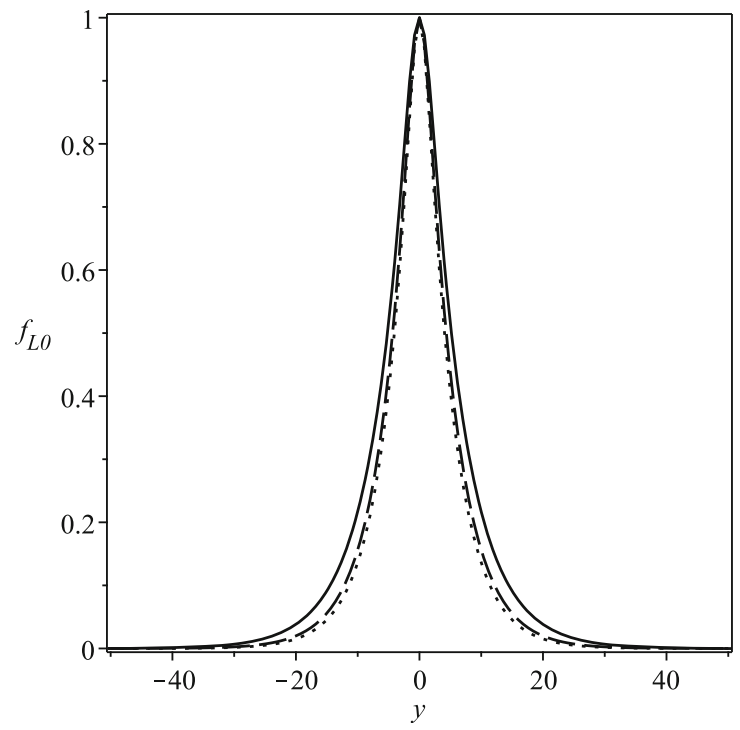

(a)

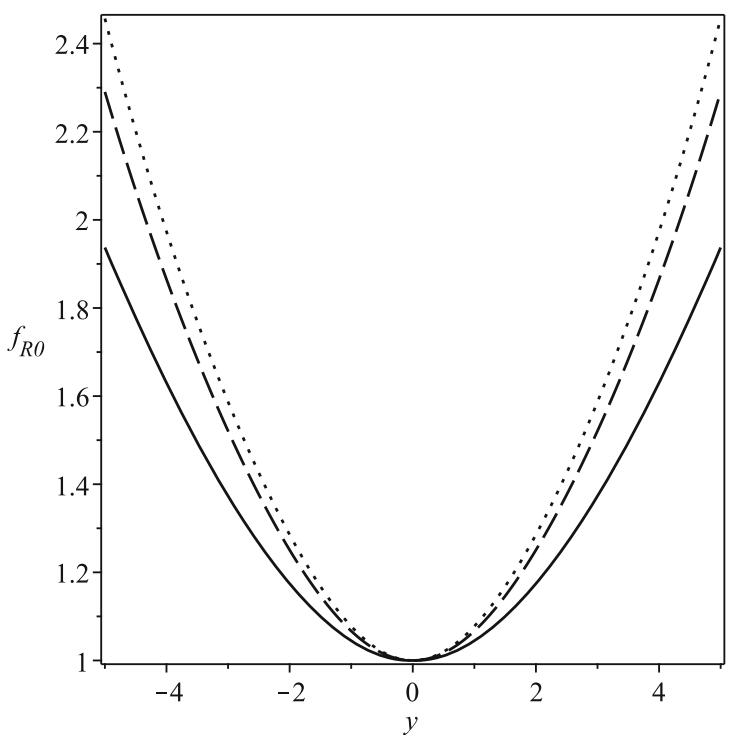

(b)

Fig. 8 The profile of $\mathbf{a}$ the left-handed zero mode and $\mathbf{b}$ the right-handed zero mode for $a=1$ (solid line), $a=2$ (dashed line), $a=5$ (dotted line), $\eta=1$

the following boundary conditions to solve the Schrodingerlike equations for fermions and similar boundary conditions for gravitons,

$f_{L n, R n}(0)=0, f_{L n, R n}^{\prime}(0)=1$, for odd $K K$ modes,

$f_{L n, R n}(0)=1, f_{L n, R n}^{\prime}(0)=0$, for even $K K$ modes.

The results of these numerical calculations are shown in Fig. 11a, b, in which the graviton and fermion massive modes are plotted in DBI braneworld models in usual gravity for $m_{n}^{2}<V_{\max }$ and $m_{n}^{2}>V_{\max }$. As we can see, the wave function oscillates quickly for $m_{n}^{2}>V_{\max }$ and reduces its period for $m_{n}^{2}<V_{\max }$. In fact it can be said that for $m_{n}^{2} \gg V_{\max }$, the wave functions of massive modes acquire the plane wave structure because the effective potential in Schrodinger-like equation represents only a small perturbation. Since we are interested in the effect of parameter $a$ on the massive modes, we again solve the Schrodinger-like equations of graviton and fermion for different values of the parameter $a$. In Figs. 12 


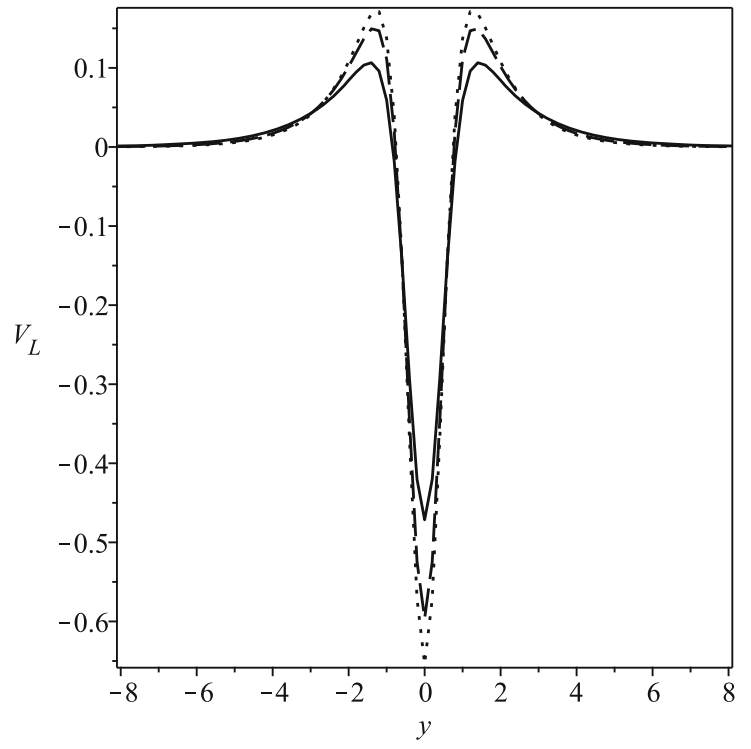

(a)

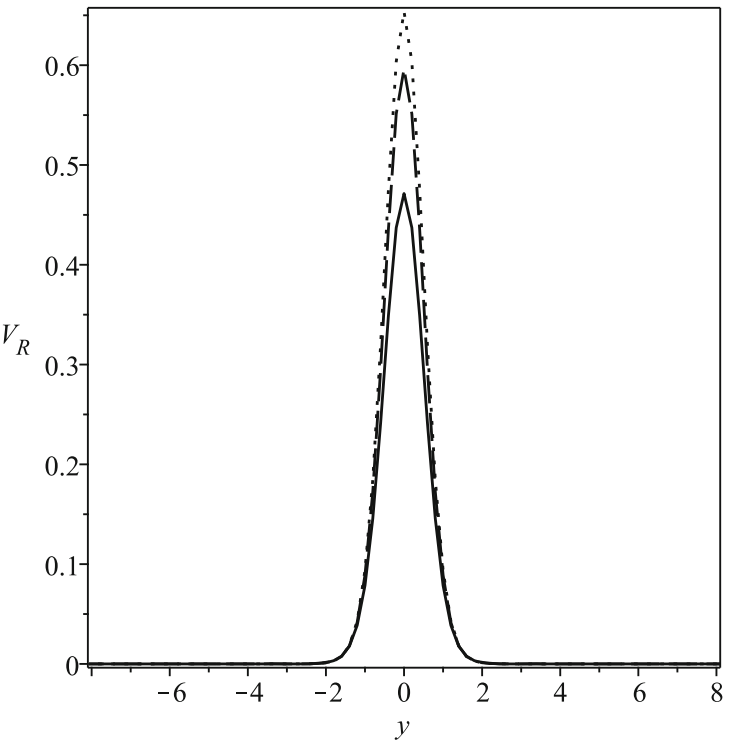

(b)

Fig. 9 The profile of the potentials a $V_{L}(y)$ and $\mathbf{b} V_{R}(y)$ for $a=1$ (solid line), $a=2$ (dashed line), $a=5$ (dotted line), $\eta=1$

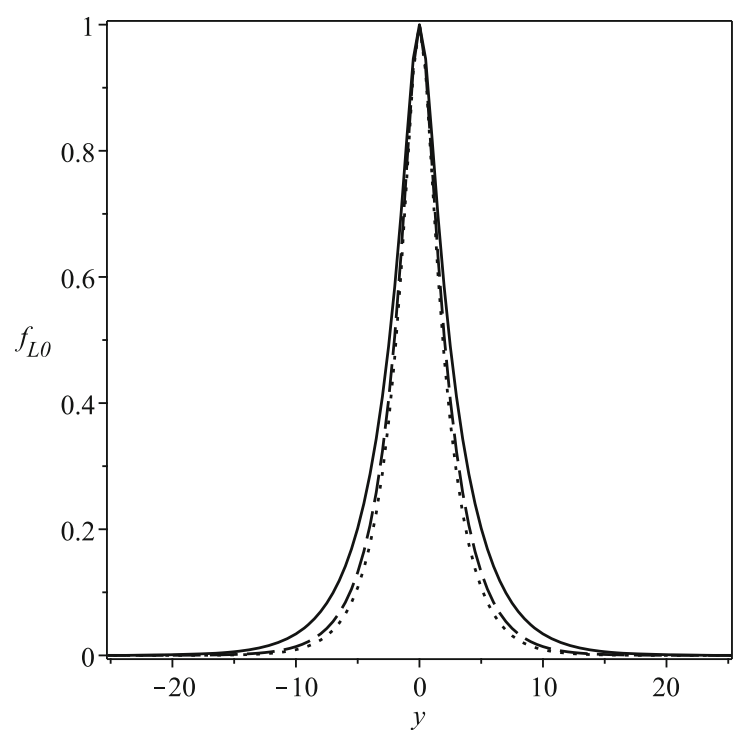

(a)

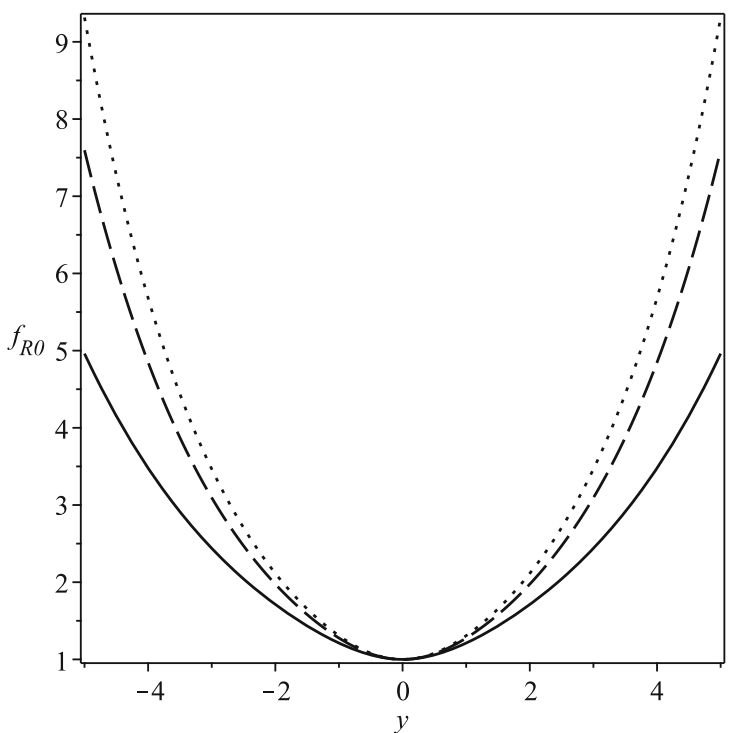

(b)

Fig. 10 The profile of $\mathbf{a}$ the left-handed zero mode and $\mathbf{b}$ the right-handed zero mode for $a=1$ (solid line), $a=2$ (dashed line), $a=5$ (dotted line), $\eta=1$

and 13, the wave functions of massive modes were plotted for DBI braneworld model in usual gravity and in $f(T)$ gravity, respectively. As Fig. 12 shows, in DBI braneworld model in usual gravity, the amplitude of the graviton massive mode as well as the period increase with increasing the parameter $a$, but in the case of fermions, the amplitude increases and the period does not change. In Fig. 13, the effect of parameter $a$ on fermion and graviton massive modes in DBI braneworld model in $f(T)$ gravity for $f_{T}=\alpha_{0}+\alpha_{1} T$ is also investi- gated. It can be seen that the graviton massive mode as well as the fermion massive mode smoothly affected by the parameter $a$ such that the amplitude and frequency of wave function increase with increasing the parameter $a$.

Finally, it should be noted that for a complete analysis of the massive modes, one must include the calculation of resonant modes. We know that in the Schrodinger-like equations such as Eqs. (48) and (49), the term $\left|f_{L, R}(z)\right|^{2}$ is described as the probability of finding the massive KK modes at the posi- 


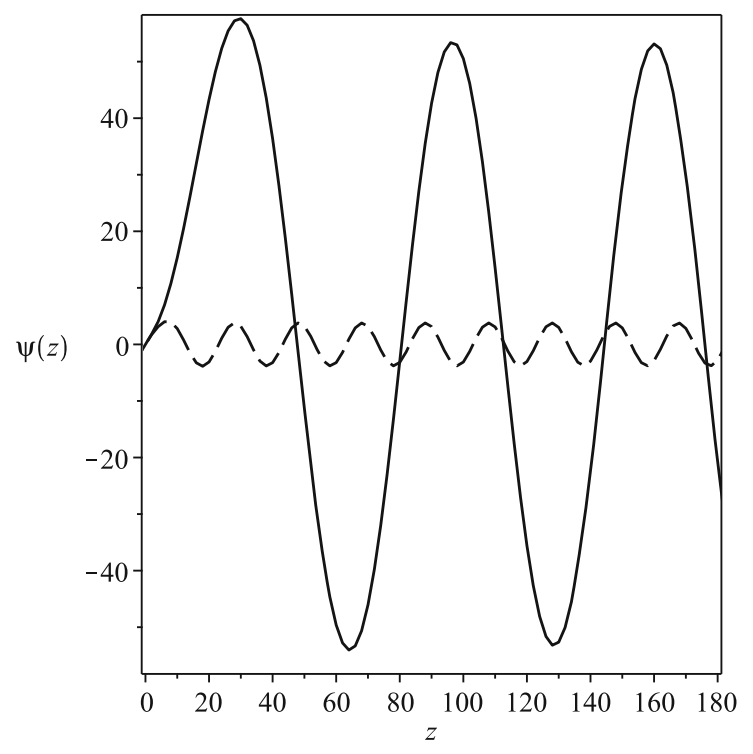

(a)

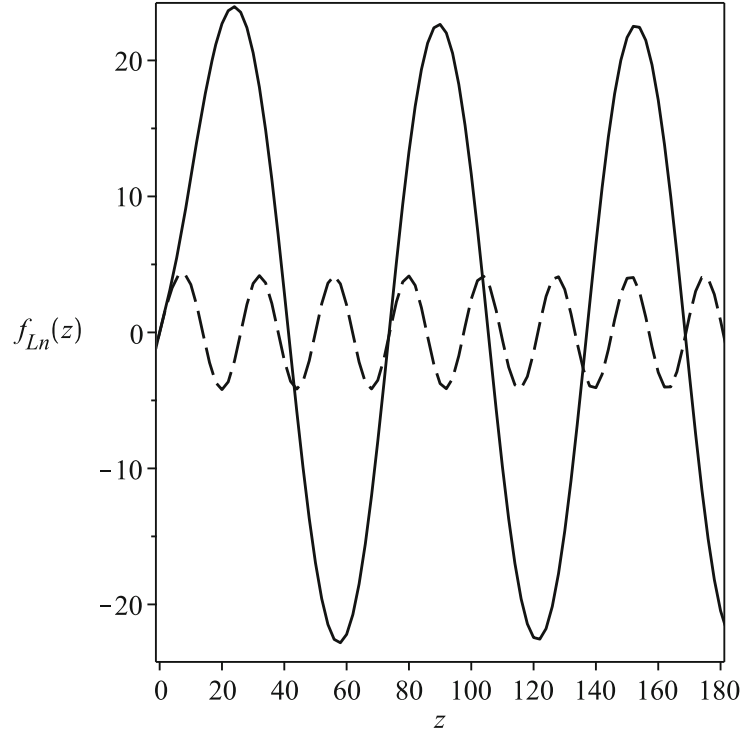

(b)

Fig. 11 The profile of a graviton wave function and $\mathbf{b}$ right-handed fermion wave function for $m_{n}^{2}<V_{\max }$ (solid line), $m_{n}^{2}>V_{\max }$ (dashed line), in all cases we have $a=5$ and $\eta=1$ for fermion case

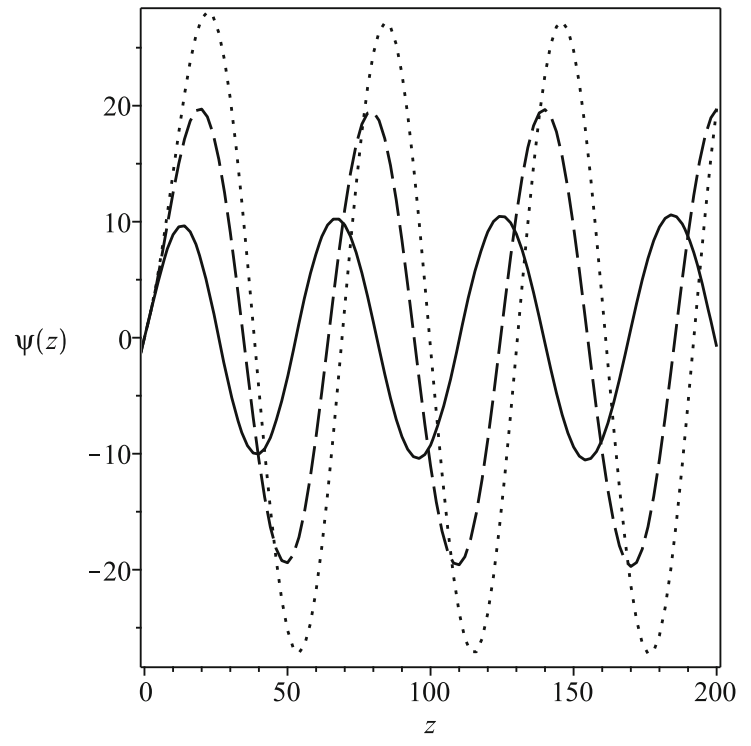

(a)

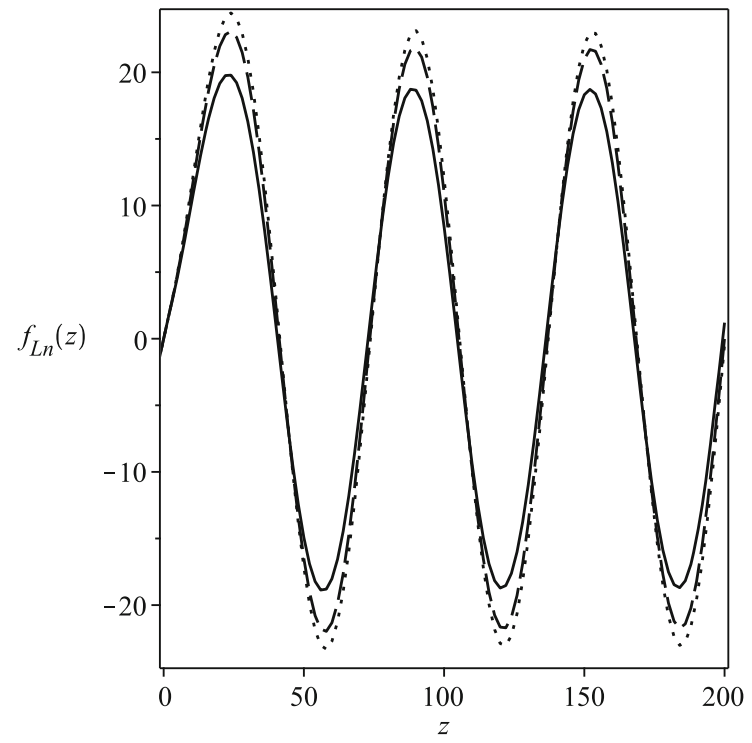

(b)

Fig. 12 The profile of a graviton wave function for $a=3$ (solid line), $a=5$ (dashed line), $a=7$ (dotted line) and $\mathbf{b}$ right-handed fermion wave function for $a=1$ (solid line), $a=2$ (dashed line), $a=5$ (dotted line), in fermion case we set $\eta=1$

tion $z$ along the extra dimension [53]. In Ref. [53], it has been suggested that large peaks in the distribution of $f_{L, R}(0)$ as a function of $m$ can represent the resonant states. But because of the condition $f_{L, R}(0)=0$ for odd parity, the authors of Ref. [54] have extended the above idea in another way. They proposed that the large relative probabilities of finding massive KK mode in a narrow range $-z_{b}<z<z_{b}$ around the brane location indicate resonant states. In our work, since the effective potentials of DBI braneworld models are not known analytically, we propose to use the relative probability method for both cases of graviton and fermion. We defer this numerical analysis for future works.

\section{Hierarchy problem in DBI braneworld models}

The hierarchy problem for braneworld models in $F(R)$ theory was studied in Ref. [37] in an interesting way. In the framework of the fermion localization and by calculating its 


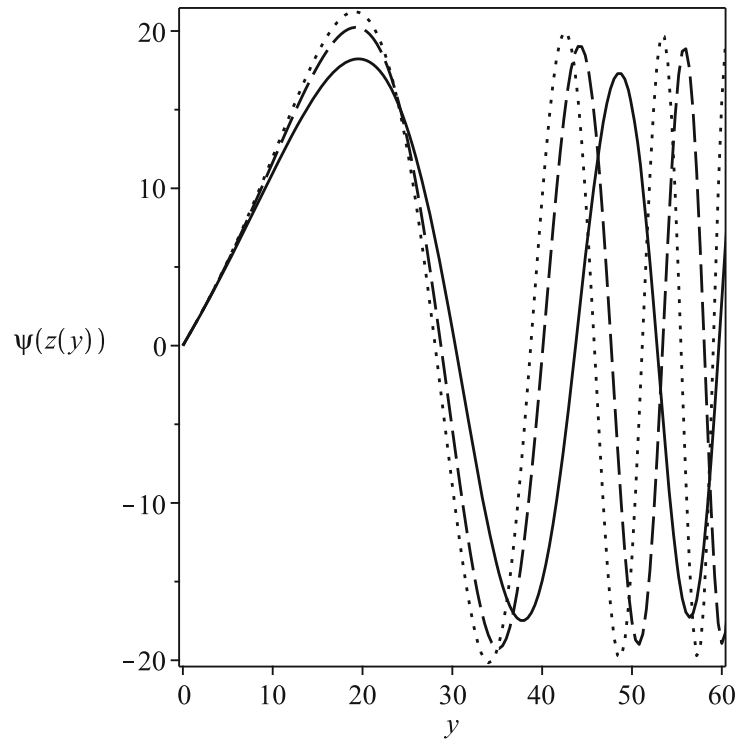

(a)

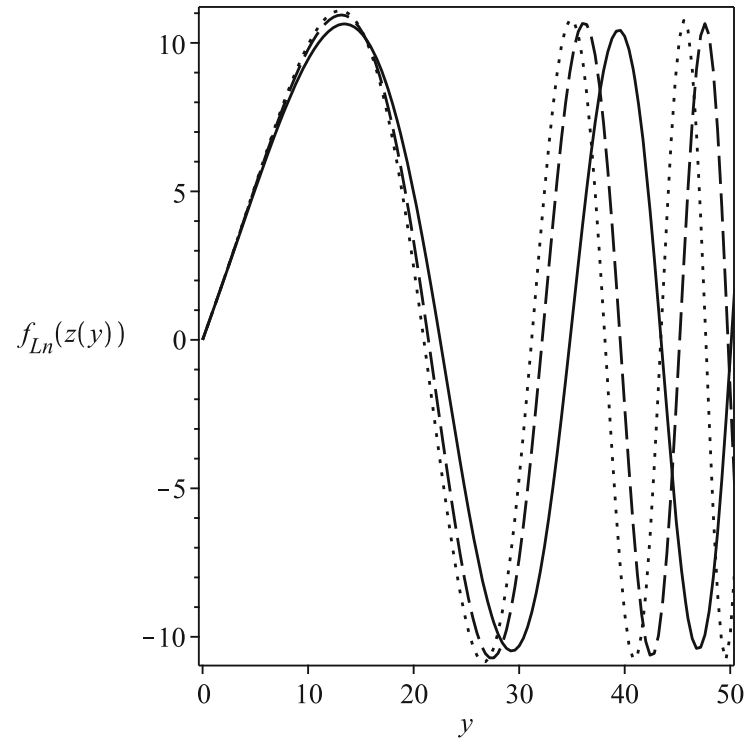

(b)

Fig. 13 The profile of a graviton wave function in $f(T)$ gravity and $\mathbf{b}$ right-handed fermion wave function in $f(T)$ gravity for $a=1$ (solid line), $a=2$ (dashed line), $a=5$ (dotted line), in all cases we set $\alpha_{0}=10$ and $\alpha_{1}=5$ and in fermion case we set $\eta=1$

mass spectrum, they showed that to solve the gauge hierarchy problem, the warp factor at Tev brane must acquire the value $=36$. As we have seen in Sect. 2, we do not have the analytical solutions of the warp factor $A(y)$ and the scalar field $\phi(y)$. So we work in a different way to solve the hierarchy problem. In this method, we first expand the kinetic energy of DBI braneworld models (9) as follows,

$K(X)=X+\frac{1}{2 a^{2}} X^{2}+\frac{1}{2 a^{4}} X^{3}+\cdots$.

Now using the above expansion we can investigate the hierarchy problem in both DBI braneworld models.

\subsection{Solving the hierarchy problem for DBI braneworld models in usual gravity}

In order to solve the hierarchy problem in DBI braneworld models in usual gravity, we put the expansion of kinetic energy (Rel. 60) in the Eq. (7) and we have

$\phi^{\prime}+\alpha \phi^{\prime 3}+O\left(\alpha^{2}\right)=\frac{1}{2} W_{\phi}$

in which we set $\alpha=\frac{-1}{2 a^{2}}$. In the following, we follow the approach outlined in Ref. [10] and to ease the investigations, we focus our study on the cases where $\alpha$ takes small values (or $a$ takes large values). So we can ignore the sentences that are of higher order $\alpha$ and write the Eq. (61) as follows,

$\phi^{\prime}+\alpha \phi^{\prime 3}=\frac{1}{2} W_{\phi}$ where the second term can be regarded as a perturbation to the standard scenario. The answer to the Eq. (62) for small values of the parameter $\alpha$ is as follows [10],

$\phi^{\prime}=\frac{1}{2} W_{\phi}-\frac{\alpha}{8} W_{\phi}$

After integrating the above equation, we have

$2 \int \frac{d \phi}{W_{\phi}}+\frac{\alpha}{2} W(\phi)=y$

From this relation we can write

$\phi(y)=\phi_{0}\left(y-\frac{\alpha}{2} W(\phi)\right)=\phi_{0}(y)-\frac{\alpha}{2} \phi_{0}^{\prime}(y) W\left(\phi_{0}(y)\right)$

where $\phi_{0}(y)$ is the solution in the standard case (for $\alpha=0$ ). Using the Eq. (63), the Eq. (65) is written as follows,

$\phi(y)=\phi_{0}(y)-\frac{\alpha}{4} W_{\phi}\left(\phi_{0}(y)\right) W\left(\phi_{0}(y)\right)$

Now according to the first order formalism and using the Eq. (6), we can write

$$
\begin{aligned}
A(y) & =-\frac{1}{3} W\left(\phi_{0}(y)-\frac{\alpha}{4} W_{\phi}\left(\phi_{0}(y)\right) W\left(\phi_{0}(y)\right)\right) \\
& =A_{0}(y)+\frac{\alpha}{12} W\left(\phi_{0}(y)\right)^{2}
\end{aligned}
$$

where $A_{0}(y)$ is the warp factor solution in the standard case (for $\alpha=0$ ). In order to obtain $A_{0}(y)$ and $\phi_{0}(y)$ in the standard case, we use the relation $\phi^{\prime}=\frac{1}{2} W_{\phi}$, and thus we have

$W_{0}^{\prime}(y)=2\left(\phi_{0}^{\prime}(y)\right)^{2}$ 
where $W_{0}(y)$ represents the superpotential function in the standard case. It can be shown that in the standard case, the function $\phi_{0}(y)$ is a function of $\tanh (y)$ and inspired by the Ref. [55], we take it in a more general form as follows,

$\phi_{0}(y)=\frac{\kappa}{\sqrt{\beta}} \tanh (\beta y)$

where $\beta$ represents the thickness regulator and $\kappa$ parametrizes the brane tension in the brane limit: $\beta \rightarrow \infty$, so that $\lambda=\frac{4}{3} \kappa^{2}$ is the brane tension. By putting the Eq. (69) into the Eq. (68) we have

$W_{0}(y)=2 \kappa^{2}\left\{\tanh (\beta y)-\frac{1}{3} \tanh ^{3}(\beta y)\right\}+W_{0 c}$

where $W_{0 c}$ is the integration constant which can be fixed by the initial condition $A^{\prime}\left(y_{\max }\right)=0$, in which $y_{\max }$ is the location of the maximum of warp factor along the extra dimension. Now, with the help of the equation $A_{0}^{\prime}(y)=-\frac{1}{3} W_{0}(y)$, we have

$A_{0}^{\prime}(y)=-\frac{2}{3} \kappa^{2}\left\{\tanh (\beta y)-\frac{1}{3} \tanh ^{3}(\beta y)\right\}-\frac{1}{3} W_{0 c}$

By integrating the above equation, we can calculate the function $A_{0}(y)$ and then we can get the function $A(y)$ as follows,

$A(y)=A_{0}(y)+\frac{\alpha}{12} W_{0}(y)^{2}$

Here we should note that in the one-brane case the hierarchy problem remains unsolved. Since our main purpose in this section is to solve the hierarchy problem, we focus on the twobrane scenario. In this scenario, the model has two branes that are located at $y=y_{1}$ and $y=y_{2}$ as UV and IR branes, respectively. So we rewrite $\phi_{0}(y)$ (Rel. (69)) as follows [55],

$\phi_{0}(y)=\frac{\kappa_{1}}{\sqrt{\beta}} \tanh \left(\beta\left(y-y_{1}\right)\right)+\frac{\kappa_{2}}{\sqrt{\beta}} \tanh \left(\beta\left(y-y_{2}\right)\right)$

where $\kappa_{1,2}$ are the brane tension parameters. As before, using the superpotential method (i.e. Eq. (68)) we can write the superpotential $W\left(\phi_{0}\right)$ as a function of $y$ as follows,

$$
\begin{aligned}
W_{0}(y)= & 2 \kappa_{1}^{2}\left\{\tanh \left(\beta\left(y-y_{1}\right)\right)-\frac{1}{3} \tanh ^{3}\left(\beta\left(y-y_{1}\right)\right)\right\} \\
& +2 \kappa_{2}^{2}\left\{\tanh \left(\beta\left(y-y_{2}\right)\right)-\frac{1}{3} \tanh ^{3}\left(\beta\left(y-y_{2}\right)\right)\right\} \\
& +W_{0 c}
\end{aligned}
$$

Therefore, the warp factor in the standard case can be written as,

$$
A_{0}^{\prime}(y)=-\frac{2}{3} \kappa_{1}^{2}\left\{\tanh \left(\beta\left(y-y_{1}\right)\right)-\frac{1}{3} \tanh ^{3}\left(\beta\left(y-y_{1}\right)\right)\right\}
$$

$$
\begin{aligned}
& -\frac{2}{3} \kappa_{2}^{2}\left\{\tanh \left(\beta\left(y-y_{2}\right)\right)-\frac{1}{3} \tanh ^{3}\left(\beta\left(y-y_{2}\right)\right)\right\} \\
& -\frac{1}{3} W_{0 c}
\end{aligned}
$$

If the branes are thin enough (or they are well separated) then the warp factor appears exponentially between them so that the hierarchy problem could be addressed. So we consider the brane limit $(\beta \rightarrow \infty)$ and we obtain all solutions including the warp factor in this limit as follows,

$$
\begin{aligned}
A_{0}^{\prime}(y)= & -\frac{1}{3}\left\{\lambda_{1} \operatorname{sgn}\left(y-y_{1}\right)+\lambda_{2} \operatorname{sgn}\left(y-y_{2}\right)\right\} \\
& +\frac{1}{3}\left\{\lambda_{1} \operatorname{sgn}\left(y_{\max }-y_{1}\right)+\lambda_{2} \operatorname{sgn}\left(y_{\max }-y_{2}\right)\right\}
\end{aligned}
$$

where $\lambda_{1,2}=\frac{4}{3} \kappa_{1,2}^{2}$ are the tensions of each brane located at $y=y_{1}$ (UV-brane) and $y=y_{2}$ (IR-brane).

In order to see the consequences of the warp background geometry and to study the hierarchy problem, we assume that the Higgs field is bounded at the IR-brane so that its action is written as follows [55],

$$
\begin{aligned}
& \text { SHiggs } \\
& \quad=-\int d^{4} x \sqrt{-g_{I R}}\left\{g_{I R}^{\mu \nu} \partial_{\mu} H^{\dagger} \partial_{\nu} H-m^{2}|H|^{2}+\lambda|H|^{4}\right\}
\end{aligned}
$$

where $m$ is the 5D Higgs mass parameter $(m \sim 5 \mathrm{D}$ Planck mass) and $g_{I R}^{\mu \nu}$ is the four dimensional metric induced on the IR brane and we have

$g_{I R}^{\mu \nu}=e^{-2 A\left(y_{2}\right)} \eta^{\mu \nu}$

where $A\left(y_{2}\right)$ is the value of warp factor at the IR-brane. Now, if we use rescaling $H \rightarrow e^{-A} H$ and the fact that $\sqrt{-g_{I R}}=$ $e^{4 A\left(y_{2}\right)}$, we arrive at

$S_{H i g g s}=-\int d^{4} x\left\{\eta^{\mu v} \partial_{\mu} H^{\dagger} \partial_{\nu} H-\mu^{2}|H|^{2}+\lambda|H|^{4}\right\}$

where $\mu=m e^{A\left(y_{2}\right)}$ is the effective Higgs mass as measured on the IR-brane. If we assume that the 5D fundamental mass scale is the Planck mass, then we can require the value of warp factor at IR-brane such that the effective 4D Higgs mass parameter is of order $\mu \sim T e v$. We suppose that the maximum value of the warp factor is at the location of UVbrane (i.e. $y_{1}=y_{\max }$ ) and so with the Eqs. (76) and (72) we have

$A\left(y_{2}\right)=-\frac{1}{3}\left(\lambda_{1}+\lambda_{2}\right) y_{2}+\frac{\alpha}{12}\left(\lambda_{1}+\lambda_{2}\right)^{2}$ 
So if we have

$$
\frac{1}{3}\left(\lambda_{1}+\lambda_{2}\right) y_{2}-\frac{\alpha}{12}\left(\lambda_{1}+\lambda_{2}\right)^{2} \sim 30
$$

then the hierarchy problem can be solved.

6.2 Solving the hierarchy problem for DBI braneworld models in $\mathrm{f}(\mathrm{T})$ gravity

In order to solve the hierarchy problem in DBI braneworld model in $f(T)$ gravity, we need to put Eq. (60) into Eq. (20) which results the following equation,

$\frac{3}{2} \partial_{y}\left(f_{T} A^{\prime}\right)=-\phi^{\prime 2}-\alpha \phi^{\prime 4}$

Now if we put the above expression equal to $-\frac{1}{2} \partial_{y} W(\phi(y))$ where $W(\phi(y))$ is a function of the scalar field $\phi(y)$, then we get the following equations,

$$
\begin{aligned}
& \frac{3}{2} \partial_{y}\left(f_{T} A^{\prime}\right)=-\frac{1}{2} \partial_{y} W(\phi(y)) \\
& -\phi^{\prime 2}-\alpha \phi^{\prime 4}=-\frac{1}{2} \partial_{y} W(\phi(y))
\end{aligned}
$$

Equations (83) and (84) are simplified as follows,

$$
\begin{aligned}
& f_{T} A^{\prime}=-\frac{1}{3} W(\phi) \\
& \phi^{\prime}+\alpha \phi^{\prime 3}=\frac{1}{2} W_{\phi}
\end{aligned}
$$

In the simplest case for $f(T)$, we can consider $f(T)=T$, so the Eq. (85) is written as follows,

$A^{\prime}=-\frac{1}{3} W(\phi)$

On the other hand, according to the Eq. (84), we can conclude that in the standard case (for $\alpha=0$ ) we have

$W_{0}^{\prime}(y)=2\left(\phi_{0}^{\prime}(y)\right)^{2}$

It can be seen that the Eqs. (86), (87) and (88) are exactly the equations that existed for solving the hierarchy problem in the first model (previous section). So all calculations in the second model for $f(T)=T$ are exactly the same as the first model, and the hierarchy problem can be addressed in the same way.

\section{Conclusions}

In this work, we have studied the localization of graviton and fermion fields in two braneworld models with nonstandard kinetic terms, which we named as (I) DBI braneworld models in usual gravity and (II) DBI braneworld models in $f(T)$ gravity. First, we consider the graviton localization in both models and we have investigated the shapes of the massindependent potentials in the corresponding schrodinger-like equations. we have found that in both models, the shapes of the effective potentials are volcano-like, which means that there is not mass gap to separate the scalar zero mode from KK modes. Meanwhile, we examined the localization conditions in both models and showed that according to the shapes of the warp factors and their behavior in infinity, these conditions always satisfied. We then obtained the zero mode of graviton in both models and we showed that in the first model, graviton is localized on the center of the brane. To do this, in the second model, we considered three special forms for $f(T)$ that were $f_{T}=\exp \left(T / T_{0}\right), f_{T}=\sum_{n=0}^{N} \alpha_{n} T^{n}$ and $f_{T}=-\ln \left(\frac{1+T}{2-T}\right)$. In each cases, we investigated the shapes of the effective potential and we found that in all three cases, they are volcano-like, but there are differences between them. For $f_{T}=\exp \left(T / T_{0}\right)$, the effective potential always has a negative well at the origin, and the zero mode of graviton is localized on the center of the brane. But for $f_{T}=-\ln \left(\frac{1+T}{2-T}\right)$, the effective potential has two negative wells on both sides of the origin and the zero mode is localized between the two sub-branes. Finally, for $f_{T}=\sum_{n=0}^{N} \alpha_{n} T^{n}$, in some values for the $\alpha$ parameters, the potential has a negative well at the origin and the zero mode is localized on the center of the brane but by changing the parameters, the negative well splits into two negative wells on both sides of the origin, so the zero mode instead of being localized on the center of brane, is localized between the two sub-branes. We also showed that in the case of $f_{T}=\sum_{n=0}^{N} \alpha_{n} T^{n}$, if we set $\alpha_{0}=1$ and the other $\alpha$ parameters are set to very small numbers so that in the extreme state, $f_{T}$ converts to $f(T)=T$, then the effective potential instead of two negative wells at the sides of the origin has a negative well at the origin and the brane splitting will disappear. This is an expected result because it is consistent with Einstein's general relativity.

After the graviton, we have investigated the fermion localization in both models. In order to localize fermions on a brane, it is necessary to introduce a Yukawa coupling between the bulk fermion field and the background scalar fields. In this paper, we have selected the Yukawa coupling as a function of the warp factor, i.e. $F(y)=-\eta \partial_{y} A(y)$. In the first model, we found that the zero mode of left-handed fermion is localized on the center of the brane. We also assumed the form of the function $f(T)$ in the second braneworld model as one of the three forms mentioned above. Under this assumption and by investigating the shapes of the mass-independent potentials of $\mathrm{KK}$ modes in the corresponding schrodingerlike equations, we have found that the potentials for each of three forms of $f_{T}$ are volcano-like and therefore the fermion field spectrum consists of a bound zero mode and a series of the continuous massive modes. On the other hand, by calcu- 
lating the localization conditions in both models, we showed that the left-chiral fermion zero mode can be localized on the branes with $F(y)=-\eta \partial_{y} A(y)$ when $\eta>\frac{1}{2}$. On the other hand, considering that the parameter $a$ is the main parameter of the DBI kinetic energy term, we were interested in studying the effect of this parameter on the graviton and fermion localization. So, throughout the paper, we plotted the graphs of the effective potentials and the zero modes for different values of parameter $a$. By examining these graphs, we found that by increasing the parameter $a$, the localization of graviton and fermion zero modes are strengthened. As a result, we can say that the zero mode of graviton and fermion are proportional to the parameter $a$, that is, as we get closer to the standard case, the localization of the zero mode of graviton and fermion are strengthened.

After thorough study of the zero mode, we performed a qualitative study of the massive KK modes. To do this, by numerical calculations we showed that for $m_{n} \gg V_{\text {max }}$, the wave function oscillates quickly and can be approximated as a plane wave. Also, numerical investigations showed that in the first model, increasing the parameter $a$ increases the amplitude and period of the wave functions of graviton and fermion. On the other hand, in the second model we consider the function $f(T)$ as $f_{T}=\alpha_{0}+\alpha_{1} T$ and we showed that by increasing the parameter $a$, both the amplitude and frequency of the wave functions of graviton and fermion increase. Of course, we need to find the resonant modes to fully investigate the massive modes. But we have postponed the study of resonant modes for future works.

Finally, due to the importance of solving the hierarchy problem in braneworld models, we study it with a perturbation method. That is, we considered non-standard kinetic energy as a perturbation to the standard scenario. Also, by considering two branes that are located at $y=y_{1}$ (UVbrane) and $y=y_{2}$ (IR-brane), it was shown that if the warp factor value at the IR-brane is of order $A\left(y_{2}\right)=$ $-\frac{1}{3}\left(\lambda_{1}+\lambda_{2}\right) y_{2}+\frac{\alpha}{12}\left(\lambda_{1}+\lambda_{2}\right)^{2} \sim-30$, then the hierarchy problem will be solved in the first DBI braneworld model. We have also shown that solving the hierarchy problem in the second DBI braneworld model can be done in a similar way, and in the particular case $f(T)=T$, the answer exactly corresponds to the answer of the first model.

Acknowledgements The authors would like to thank the unknown referee for the positive feedback and useful comments.

Data Availability Statement This manuscript has no associated data or the data will not be deposited. [Authors' comment: There is no associated data.]

Open Access This article is licensed under a Creative Commons Attribution 4.0 International License, which permits use, sharing, adaptation, distribution and reproduction in any medium or format, as long as you give appropriate credit to the original author(s) and the source, provide a link to the Creative Commons licence, and indicate if changes were made. The images or other third party material in this article are included in the article's Creative Commons licence, unless indicated otherwise in a credit line to the material. If material is not included in the article's Creative Commons licence and your intended use is not permitted by statutory regulation or exceeds the permitted use, you will need to obtain permission directly from the copyright holder. To view a copy of this licence, visit http://creativecomm ons.org/licenses/by/4.0/.

Funded by $\mathrm{SCOAP}^{3}$.

\section{References}

1. V.A. Rubakov, M.E. Shaposhnikov, Phys. Lett. B 125, 139 (1983)

2. R. Linares, H.A. Morales-Tecotl, O. Pedraza, Phys. Rev. D 81, $126013(2010)$

3. N. Arkani-Hamed, S. Dimopoulos, G. Dvali, Phys. Lett. B 429, 263 (1998)

4. L. Randall, R. Sundrum, Phys. Rev. Lett. 83, 3370 (1999)

5. W.D. Goldberger, M.B. Wise, Phys. Rev. Lett. 83, 4922 (1999)

6. A. Dey, D. Maity, S. Sengupta, Phys. Rev. D 75, 107901 (2007)

7. A. Tofighi, M. Moazzen, Mod. Phys. Lett. A 28, 1350044 (2013)

8. L.N. Granda, A. Oliveros, Europhys. Lett. 74, 236 (2006)

9. A. Kehagias, K. Tamvakis, Phys. Lett. B 504, 38 (2001)

10. D. Bazeia, A.R. Gomes, L. Losano, R. Menezes, Phys. Lett. B 671, 402 (2009)

11. D. Bazeia, F.A. Brito, J.R.S. Nascimento, Phys. Rev. D 68, 085007 (2003)

12. R. Koley, S. Kar, Phys. Lett. B 623, 244 (2005)

13. C. Adam, N. Grandi, P. Klimas, J. Sanchez-Guillen, A. Wereszczynski, J. Phys. A 41, 375401 (2008)

14. Y.-X. Liu, Y. Zhong, K. Yang, Europhys. Lett. 90, 51001 (2010)

15. L.B. Castro, L.A. Meza, Europhys. Lett. 102, 21001 (2013)

16. E. Silverstein, D. Tong, Phys. Rev. D 70, 103505 (2004)

17. M. Alishahiha, E. Silverstein, D. Tong, Phys. Rev. D 70, 123505 (2004)

18. X. Chen, Phys. Rev. D 71, 063506 (2005)

19. S. Weinberg, Phys. Rev. D 77, 123541 (2008)

20. D. Bazeia, E.E. Lima, L. Losano, Ann. Phys. 388, 408 (2018)

21. B. Bajc, G. Gabadadze, Phys. Lett. B 474, 282 (2000)

22. H. Guo, Y.-X. Liu, S.-W. Wei, C.-E. Fu, Euro phys. Lett 97, 60003 (2012)

23. A. Tofighi, M. Moazzen, Acta Phys. Polon. b 45, 1797 (2014)

24. M. Moazzen, Z. Ghalenovi, Ann. Phys. 385, 70 (2017)

25. A. Neronov, Phys. Rev. D 64, 044018 (2001)

26. H. Christiansen, M. Cunha, Eur. Phys. J. C 72, 1942 (2012)

27. C.A. Vaquera-Araujo, O. Corradini, Eur. Phys. J. C 75, 48 (2015)

28. R. Cartas-Fuentevilla, A. Escalante, G. German, A. HerreraAguilar, R.R. Mora- Luna, JCAP 1605, 026 (2016)

29. A. Tofighi, M. Moazzen, Int. J. Theor. Phys. 50, 1709 (2011)

30. A. Tofighi, M. Moazzen, Int. J. Mod. Phys. A 29(24), 1450126 (2014)

31. Y.-X. Liu, Z.-G. Xu, F.-W. Chen, S.-W. Wei, Phys. Rev. D 89, 086001 (2014)

32. Y.-Y. Li, Y.-P. Zhang, W.-D. Guo, Y.-X. Liu, Phys. Rev. D 95, 115003 (2017)

33. Y.-X. Liu, H. Guo, C.-E. Fu, H.-T. Li, Phys. Rev. D 84, 044033 (2011)

34. M. Moazzen, Int. J. Mod. Phys. A 32, 1750058 (2017)

35. R.C. Fuentevilla, A. Escalante, G. German, A. Herrera-Aguilara, R. Rigel Mora-Luna, JCAP 05, 026 (2016)

36. J. Mitra, T. Paul, S. SenGupta, Eur. Phys. J. C 77, 833 (2017)

37. T. Paul, S. SenGupta, Phys. Rev. D 95, 115011 (2017)

38. J. Yang, Y.-L. Li, Y. Zhong, Y. Li, Phys. Rev. D 85, 084033 (2012)

39. R. Menezes, Phys. Rev. D 89, 125007 (2014) 
40. W.-D. Guo, Q.-M. Fu, Y.-P. Zhang, Y.-X. Liu, Phys. Rev. D 93, 044002 (2016)

41. J. Wang, W.D. Guo, Z.C. Lin, Y.X. Liu, Phys. Rev. D 98, 084046 (2018)

42. P. Pedram, Europhys. Lett. 89, 50008 (2010)

43. N. Evans, J. French, K.Y. Kim, J. High Energ. Phys. 145, 1011 (2010)

44. R. Gwyn, M. Sakellariadou, S. Sypsas, J. High Energ. Phys. 1010, 075 (2010)

45. K. Skenderis, P.K. Townsend, Phys. Lett. B 468, 46 (1999)

46. B. Born, L. Infeld, Proc. R. Soc. A 144, 425 (1935)

47. P.A.M. Dirac, Proc. R. Soc. A 268, 57 (1962)

48. H. Guo, A. Herrera-Aguilar, Y.X. Liu, D. Malagon-Morejon, R.R. Mora-Luna, Phys. Rev. D 87, 095011 (2013)

49. I. Oda, Phys. Rev. D 64, 026002 (2001)
50. W.T. Cruz, M.O. Tahim, C.A.S. Almeida, EPL (Europhysics Letters) 88, 41001 (2009)

51. W.T. Cruz, R.V. Maluf, D.M. Dantas, C.A.S. Almeida, Ann. Phys. 375 (2016)

52. M. Moazzen, Int. J. Mod. Phys. A 32, 1750058 (2017)

53. C.A.S. Almeida, R. Casana, M.M. Ferreira Jr., A.R. Gomes, Phys. Rev. D 79, 125022 (2009)

54. Y.X. Liu, J. Yang, Z.H. Zhao, C.E. Fu, Y.S. Duan, Phys. Rev. D 80, 065019 (2009)

55. A. Ahmed, B. Grzadkowski, JHEP 177, 1301 (2013) 